\title{
Estimating the Readily-Releasable Vesicle Pool Size at Synaptic Connections in a Neocortical Microcircuit
}

\author{
${ }^{1}$ Barros-Zulaica N, ${ }^{1}$ Rahmon $\mathrm{J},{ }^{1} \mathrm{Chindemi}$ G, ${ }^{2}$ Perin $\mathrm{R},{ }^{1,2}$ Markram $\mathrm{H},{ }^{1}$ Ramaswamy $\mathrm{S}$ and \\ ${ }^{1}$ Muller E
}

${ }^{1}$ Blue Brain Project, École polytechnique fédérale de Lausanne, Campus Biotech, 1202 Geneva, Switzerland

${ }^{2}$ Blue Brain Project, École polytechnique fédérale de Lausanne, LNMC, 1015 Lausanne, Switzerland

\section{* Correspondence:}

Natalí Barros Zulaica

natali.barroszulaica@epfl.ch

Keywords: synaptic transmission, quantal analysis, multi vesicular release, neocortex, mathematical model, short-term depression.

\begin{abstract}
Previous studies based on the 'Quantal Model' for synaptic transmission suggested that neurotransmitter release is mediated by a single release site at individual synaptic contacts in the neocortex. However, recent studies seem to contradict this hypothesis and indicate that multi-vesicular release (MVR) could better explain the synaptic response variability observed in vitro. In this study we present a novel method to estimate the number of release sites per synapse, also known as the size of the readily-releasable pool ( $\mathrm{N}_{\mathrm{RRP}}$ ), from paired whole-cell recordings of layer 5 thick tufted pyramidal cell (L5_TTPC) connections in the somatosensory neocortex. Our approach extends the work of Loebel and colleagues to take advantage of a recently reported data-driven biophysical model of neocortical tissue. Using this approach, we estimated $\mathrm{N}_{\mathrm{RRP}}$ to be between two to three for connections between L5-

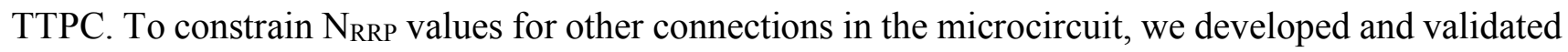
a generalization approach using data on post-synaptic potential (PSP) coefficient of variations (CVs) from literature and matching to in silico experiments. Our study shows that synaptic connections in the neocortex generally are mediated by MVR and provides a data-driven approach to constrain the MVR model parameters of the microcircuit.
\end{abstract}




\section{Introduction}

Synaptic transmission is the basis for neuronal communication and information processing in the brain. Synaptic communication between neurons is mediated by neurotransmitters contained in presynaptic vesicles that are stochastically released from axonal boutons by incoming action potentials (APs) and diffuse across the synaptic cleft to bind receptors. Synaptic receptors are a class of ion channels which open as a result of transmitter binding, and the resulting transmembrane currents either depolarize or hyperpolarize the postsynaptic membrane, depending on the ion to which the channel is permeable (Mason et al., 1991; Südhof, 2000). Understanding the mechanisms behind vesicle release is crucial to unravel how information propagates between different types of neurons (Tsodyks and Markram, 1997). In fact, disruptions in vesicle release are implicated in pathologies such as Alzheimer's disease or schizophrenia (Waites and Garner, 2011).

In 1954, del Castillo and Katz described the 'Quantal model' of synaptic transmission (Del Castillo and Katz, 1954). This model is characterized by the number of independent release sites (N), the probability of releasing a vesicle in the presynaptic cell followed by an AP (p) and the content of each vesicle, the quantal size (q), which collectively determine the efficacy of synaptic transmission (Del Castillo and Katz, 1954; Tsodyks and Markram, 1997). Previously, it was thought that no more than one vesicle could be released per synaptic contact, leading to the univesicular release hypothesis (UVR), in which $\mathrm{N}$ is equal to the number of physical synaptic contacts in a neuronal connection, at least for synapses in the neocortex (Biró et al., 2005; Korn et al., 1981, 1994; Silver et al., 2003). However, evidences as fluctuations of evoked post-synaptic potentials (PSPs) (Tang et al., 1994), large concentration of neurotransmitter in the synaptic cleft (Tong and Jahr, 1994) or a high range variability of receptor-mediated signals of N-methyl-D-aspartate (NMDA) and $\alpha$-amino-3-hydroxy-5-methyl-4isoxazolepropionic acid (AMPA) receptors (Conti and Lisman, 2003) suggested that transmission at a single synaptic contact could be multiquantal. Consequently, a multivesicular release hypothesis (MVR) was proposed, where several release sites could underlie a synaptic contact in a neuronal connection. In fact, there are evidences showing that MVR occurs in brain regions such as the hippocampus (Christie and Jahr, 2006; Tong and Jahr, 1994), the cerebellum (Auger et al., 1998), the hypothalamus (Gordon, 2005) or the cerebral cortex (Huang et al., 2010; Molnár et al., 2016; Rudolph et al., 2015). Furthermore, MVR has been physically observed in individual contact sites of the mouse hippocampus with 'flash-and-freeze' electron microscopy technique (Watanabe et al., 2013). 
In the neocortex, recent studies in rodents support the idea of MVR between pyramidal cells (Hardingham et al., 2010; Loebel et al., 2009; Rollenhagen et al., 2018). It has also been reported that different cortical areas show different vesicular release behavior. For instance, it has been reported that connections between cells in layer 4 exhibit UVR in the primary visual cortex, but the primary somatosensory cortex exhibit MVR (Huang et al., 2010). By contrast, other studies have reported that connections between layer 4 stellate cells and layer $2 / 3$ pyramidal cells in the rat barrel cortex (Silver et al., 2003) and between pyramidal cells and interneurons in the rat cortex (Molnár et al., 2016) follow the UVR hypothesis. Moreover, in human neocortex, MVR has been observed for connections between pyramidal cells and fast spiking interneurons (Molnár et al., 2016).

Theoretical models have also contributed to understand vesicular release by studying synaptic processes as short-term synaptic plasticity. These models account for crucial parameters for modeling the presynaptic release such as the number of vesicles available for release and the probability of releasing these vesicles (Hennig, 2013; Loebel et al., 2009; Tsodyks and Markram, 1997). These models also assume that the rate of releasable neurotransmitter is limited and they take into account vesicle depletion (Liley and North, 1953), facilitation mechanisms (Betz, 1970; Markram et al., 1998; Varela et al., 1997) and vesicle replenishment (Fuhrmann et al., 2004). Many models has reported evidences of the importance in the number of release sites per neuronal connection for instance for information coding (Fuhrmann et al., 2002). It has also been reported that the frequency in which multiple vesicles are released and the number of vesicles released is very important for receptor activation (Boucher et al., 2010). Some studies also reveal the importance of having the number of readily releasable pool ( $\mathrm{N}_{\mathrm{RRP}}$ ) higher than 1 for being able to reproduce plasticity processes (Nadkarni et al., 2010). $\mathrm{N}_{\mathrm{RRP}}$ represents the number of docked vesicles ready to be released at boutons of the presynaptic axon when an action potential is propagating. Other works found evidences of MVR at different synapses (Loebel et al., 2009) using synaptic models.

As MVR is a complex process that is suspected to regulate synaptic transmission and plasticity by increasing the dynamic range of synapses (Fuhrmann et al., 2004) influencing cognitive functions such as learning and memory. MVR is thought to directly impact synaptic noise and variability, with larger vesicle pool sizes resulting in a reduction of synaptic noise, with important implications for the transmission of information (Fuhrmann et al., 2004). Thus, accurate models of synaptic transmission in the neocortical microcircuit (Markram et al., 2015) must therefore account for the current state of knowledge on MVR. 
Taken together, these previous studies demonstrate the complexity of the vesicle release process in the cortex, and show that there remains a need for developing an integrated consensus on vesicle pool sizes in the rodent neocortex.

In this work, we estimated the average size of the $\mathrm{N}_{\mathrm{RRP}}$ for individual synaptic contacts between different cell types in the neocortical microcircuit. To compute this parameter, we employed datadriven simulations of connected pairs of neurons (Markram et al., 2015) with previous reported counterpart in vitro experiments, taking care to reproduce pertinent aspects of the experiments such as the spatial distributions of neuron sampling, specific synaptic parameters and sub-threshold noise sources. We then optimized model MVR parameters, in particular $\mathrm{N}_{\mathrm{RRP}}$, to reproduce response variability as observed in the experiments, as assessed by coefficients of variation (CVs) of excitatory postsynaptic potentials (EPSPs). We first made use of a high quality in vitro dataset on synaptic connections between layer 5 thick-tufted pyramidal cells (L5_TTPC) in the juvenile rat somatosensory cortex to estimate synaptic and noise model validity and the MVR free parameter $\mathrm{N}_{\mathrm{R} R \mathrm{P}}$, extending the work of Loebel and colleges (Loebel et al., 2009) to morphologically detailed neuron models with anatomically and physiologically constrained connectomes. We further developed an approach to estimate $\mathrm{N}_{\mathrm{RRP}}$ for both excitatory and inhibitory connection types using literature data sources providing PSP CVs. Our work provides evidence for MVR at most of the connection types examined, albeit with lower $\mathrm{N}_{\text {RRP }}$ values than previously reported by Loebel et al., 2009, suggesting it could be a general property of local cortical connections.

\section{Materials and Methods}

\subsection{Slice preparation and electrophysiology}

Fourteen- to eighteen-day-old Wistar rats were quickly decapitated according to the Swiss Welfare Act and the Swiss National Institutional Guidelines on Animal Experimentation for the ethical use of animals. The project was approved by the Swiss Cantonal Veterinary office following its ethical review by the State Committee for Animal Experimentation. Multiple (6-12 cells simultaneously) somatic whole cell patch-clamp recordings were carried out with Multiclamp 700B amplifiers in current clamp mode. Brain sagittal slices of $300 \mu \mathrm{M}$ width were cut on an HR2 vibratome (Sigmann Elektronik). Temperature was held at $34 \pm 1{ }^{\circ} \mathrm{C}$ in all experiments. The extracellular solution contained $125 \mathrm{mM}$ $\mathrm{NaCl}, 2.5 \mathrm{mM} \mathrm{KCl}, 25 \mathrm{mM}$ D-glucose, $25 \mathrm{mM} \mathrm{NaHCO} 3,1.25 \mathrm{mM} \mathrm{NaH} 2 \mathrm{PO} 4,2 \mathrm{mM} \mathrm{CaCl}$, and 1 
$\mathrm{mM} \mathrm{MgCl} 2$ bubbled with 95\% O2 and 5\% CO2. The intracellular pipette solution contained $110 \mathrm{mM}$ potassium gluconate, $10 \mathrm{mM} \mathrm{KCl}, 4 \mathrm{mM}$ ATP-Mg, $10 \mathrm{mM}$ phosphocreatine, $0.3 \mathrm{mM} \mathrm{GTP}, 10$ Hepes, and $13 \mathrm{mM}$ biocytin adjusted to $\mathrm{pH} 7.3-7.4$ with $5 \mathrm{M} \mathrm{KOH}$.

Data was acquired using PulseQ, a custom-writen data acquisition library written in IGOR (WaveMetrics, Lake Oswego, OR). L5_TTPCs were selected according to their large soma size (15$25 \mu \mathrm{m})$ and their apparent large trunk of the apical dendrite. Cells were visualized by infrared differential interference contrast video microscopy using a VX55 camera (Till Photonics) mounted on an upright BX51WI microscope (Olympus). Data acquisition was performed through an ITC-1600 board (Instrutech) connected to a PC running a custom-written routine (PulseQ) under IGOR Pro (Wavemetrics). Sampling rates were $5-10 \mathrm{kHz}$, and the voltage signal was filtered with a 2-kHz Bessel filter. The stimulation protocol consisted of pre-synaptic stimulation with eight electric pulses at $20 \mathrm{~Hz}$ followed by a single pulse $500 \mathrm{~ms}$ later (recovery test), at the sufficient current intensity to generate APs in the presynaptic neuron while the postsynaptic neuron responses were recorded. The protocol was repeated from 20 to 60 times. (Fig.1A up)

\subsection{Stochastic model for short-term dynamics and multi-vesicular release}

Our model describes the short-term synaptic dynamics defined by a stochastic generalization of the Tsodyks-Markram model (TM-model) (Maass and Markram, 2002; Tsodyks and Markram, 1997) that is known to fit excitatory as well as inhibitory synapses behavior of biological experiments (Gupta et al., 2000; Markram et al., 1998). This model considers that there is a finite number of vesicles ready to be released defined by $\mathrm{N}_{\mathrm{RRP}}$ that could be in ready or recovery state. In this study we followed the synaptic dynamics described on Maass and Markram, 2002 that is able to predict the sequence of postsynaptic potential (PSP) amplitudes produced by any spike train. This behavior is described by four main synaptic parameters: the absolute synaptic efficacy (A), the fraction of synaptic resources used by a single spike (U), the time constant for recovery from facilitation (F) and the time constant for recovery from depression (D). The PSP amplitudes prediction obeys the following mathematical expressions:

$$
\begin{gathered}
A_{n}=A u_{n} R_{n} \\
A=1
\end{gathered}
$$




$$
\begin{gathered}
u_{1}=U \\
R_{1}=1 \\
u_{n+1}=U+u_{n}(1-U) \exp \left(-\frac{\Delta t_{n}}{F}\right) \\
R_{n+1}=1+\left(R_{n}-R_{n} u_{n}-1\right) \exp \left(-\frac{\Delta t_{n}}{D}\right)
\end{gathered}
$$

In short, when the nth spike occurs there is certain fraction of synaptic efficacy modeled by $\mathrm{R}_{\mathrm{n}}$. Accordingly, the product $u_{n} R_{n}$ models the fraction of synaptic efficacy used by the nth spike. Combining these terms is possible to describe the fraction of synaptic efficacy available when the next spike arrives at time $\Delta \mathrm{t}_{\mathrm{n}}$ assuming that the synaptic efficacy has an exponential recovery with time constant D. How much fraction of synaptic efficacy $\left(R_{n+1}\right)$ is used when $(n+1)$ th spike occurs is defined by $u_{n+1}$ which increases for each subsequent spike from $u_{n}$ to $U\left(1-u_{n}\right)+u_{n}$ and goes back to $U$ following an exponential with time constant F (Maass and Markram, 2002).

Our model also takes into consideration postsynaptic models for AMPA and NMDA receptors. Thus, if a vesicle is successfully released, these receptors get activated with a conductance $g_{\max } / \mathrm{N}_{\text {RRP }}$ with $\mathrm{g}_{\max }$ as the maximal conductance.

\subsection{Fitting synapse model parameters to the data}

We constrained our model by extracting the parameters $\mathrm{U}, \mathrm{D}$ and $\mathrm{F}$ from each in vitro connection (Fig.2C, D and E). For this purpose, we needed to compute the EPSP amplitudes of each averaged voltage trace. All experimental traces were normalized to their maximum value, so the maximum amplitude would be 1 and we could directly compute the peak value instead of the total amplitude. To perform an accurate computation of the peaks we used a mathematical tool for deconvolving the voltage averaged trace (Richardson and Silberberg, 2008). Basically, what this method provided us was the possibility of removing the smoothing effect of the membrane cell low-pass filter with a time scale equal to $\tau_{\mathrm{mem}}$, so we could extract the peaks from the EPSPs, all of them leveled to the same voltage base (Fig.2B).

To express this process mathematically we used the next equation:

$$
R_{\text {input }} I_{\text {syn }}=\tau_{\text {mem }} \frac{d V}{d t}+V
$$


The right-hand part of the expression is the voltage deconvolution, while the left hand contains the unfiltered synaptic current. The requirement here is to compute $\tau_{\text {mem }}$ for each in vitro connection by fitting the decay part of the recovery peak $\left(9^{\text {th }}\right.$ EPSP) of the averaged voltage trace to an exponential.

Once we got the peaks, we matched the possible U, D and F parameters with the help of a genetic algorithm (Goldberg and Holland, 1988) with 500 generations. Using the mathematical expression of the model, the input values were the peaks of each trace and the ranges for $U, D$ and $F$ were $(0,1.0)$, $(0,1000.0)$ and $(0,2000.0)$ respectively. We obtained one set of $U, D$ and $F$ per connection and consequently we defined these parameters as distributions (normal, gamma and gamma respectively) with their correspondent mean and standard deviation.

\subsection{In Silico experiments: the cortical microcircuit.}

For the in silico experiments we took advantage of the somatosensory cortical microcircuit built into the frame of the Blue Brain Project (Markram et al., 2015). This is a data driven model with detailed anatomy and physiology with 31000 neurons, 8 million connections and 37 million synapses in a volume of $0.29 \mathrm{~mm}^{3}$.

Once we got the values for the mean and the standard deviation of the synaptic parameters from the fitting of the in vitro data to the TM-model, we updated the simulation with these new values implemented as distribution defined by their mean and standard deviation. We also computed $\mathrm{g}_{\max }$ through the simulation of synaptic connections with different $g_{\max }$ values and we kept the $g_{\max }$ values which first EPSP amplitude was similar to the real data. After all the updates, we were able to perform patch-clamp in silico experiments (Fig1.B up), with the same conditions as the in vitro, with different $\mathrm{N}_{\mathrm{RRP}}$ values. These values were shaped defining the mean of a Poisson distribution of 100 points shifted one unit to the right. The range of means of the poissonian distributions varied from 0 to $13\left(1 \leq \mathrm{N}_{\mathrm{RRP}}\right.$ $\geq 14)$ in the case of studying MVR and $0\left(\mathrm{~N}_{\mathrm{RRP}}=1\right)$ while studying UVR. We decided to set the maximum value to 14 vesicles on average per release site because is already the double of what Loebel and colleges predicted on their research (Loebel et al., 2009).

Subsequently, we run simulations for 100 L5_TTPC connections of the microcolumn with 20 protocol repetitions. As these simulations will be used to be compare with the in vitro traces, we had to be sure that $\mathrm{U}, \mathrm{D}$ and $\mathrm{F}$ distributions for the in silico connections had the same shape as for the in vitro. To this end we set the distributions before running the simulations. After running the simulations, we checked 
that the $1^{\text {st }}$ EPSP amplitude for the simulations were within the range of the in vitro data collection. In the case of UVR study we didn't have to remove any experiment, but in the case of MVR we removed 15 connections (85 out of 100) according to the criteria explained before.

\subsection{Noise calibration. Ornstein-Uhlenbeck process}

After performing the simulations with different $\mathrm{N}_{\text {RRP }}$ values and selecting the simulations within the $1^{\text {st }}$ EPSP amplitude range of the in vitro connections, we had to complete the simulated traces with voltage fluctuations that will account for the membrane noise. One way of doing it was implementing the Ornstein-Uhlenbeck process (OU-process), which is a stochastic process that allowed us to simulate small random variability. The OU-process describes the velocity of the movement of a Brownian particle considering the friction and is a stationary Gauss-Markov process (Enrico Bibbona, 2008).

Mathematically the expression used in this work for this process was:

$$
\begin{gathered}
X(t+1)=X(t)-\frac{X(t)}{\tau} d t+\sigma \sqrt{\frac{2}{\tau}} W_{t} \\
X\left(t_{0}\right)=x_{0}
\end{gathered}
$$

Where $\tau$ is the membrane time constant, $\sigma$ is the standard deviation of the voltage and $\mathrm{W}_{\mathrm{t}}$ is a random term coming from the Wiener process. In the case of $\sigma=0$ the equation will have the solution $X(t)=$ $x_{0} e^{-\left(t-t_{0}\right) / \tau}$ so $\mathrm{X}(\mathrm{t})$ relaxes exponentially towards 0 . In general, $\mathrm{X}(\mathrm{t})$ fluctuates randomly, the third term pushes it away from zero, while the second term pulls it back to zero (Bradley Efron and Robert J. Tibshirani, 1994). In Physics this process is used to describe noisy relaxation activity.

In our specific case we defined $\sigma$ and $\tau$ using the voltage values between the 8 th and the 9th EPSPs, $400 \mathrm{~ms}$ in total, for each repetition (sweep) in a connection and then we averaged the resulting values (Fig.3A). By computing the standard deviation of these points, we got one $\sigma$ per connection (33 in total). Through the calculation of the autocorrelation of this section and fitting it to an exponential, we got one $\tau$ per connection (Fig.3B). The computation of their means gave us the values to implement the membrane noise for the in silico traces (Fig.3C). 


\subsection{CV profile computation. The Jack-Knife bootstrapping analysis}

In order to compute the $\mathrm{CV}$ for the EPSP amplitudes in the case of the in vitro and the in silico experiments in the more accurate possible way, we implemented the Jack-Knife method (JKK) (Bradley Efron and Robert J. Tibshirani, 1994).

This method consists in excluding one observation at a time from a group of observations. In our specific case, from a set of single traces we computed the average of all but one off the traces each time, obtaining at the end a set of averaged-JKK traces. From each of this averaged-JKK traces we computed the amplitudes for the nine EPSPs. This computation is much more precise considering we removed the noise by averaging. Then we could compute the CV profiles for the in vitro and the in silico experiments using the following equations:

$$
\begin{gathered}
C V^{n}=\frac{s t d^{n}}{\bar{A}^{n}} \\
\bar{A}^{n}=\frac{1}{N} \sum_{i=1}^{N} A_{i}^{n} \\
s t d^{n}=\sqrt{(N-1) \sum_{i=1}^{n}\left(A_{i}^{n}-\bar{A}_{i}^{n}\right)}
\end{gathered}
$$

Where $n$ denotes the EPSP index $(n=1, \ldots, 9)$ and $\mathrm{N}$ is the number of single traces per connection.

Once having both simulation sets, to study UVR and MVR, we could compute the CV profile for the EPSP amplitudes using the JKK approach in both cases and compare them with the CV profile computed with the in vitro data. We computed the mean square distance in order to obtain the minimum error between in vitro and in silico CV profiles (Fig. 4E). We iterated this procedure 50 times and then we provided the mean and the standard deviation for the $\mathrm{N}_{R R P}$ that correspond with the smallest error.

\section{Results}

\subsection{Motivation for implementing MVR in the model}

Our simulation was based on a biologically detailed, data-driven model of a rat neocortical microcircuit (Markram et al., 2015). Although this data-driven simulation was capable of reproducing many biological results, some improvements were needed. For instance, our goal in this work, which was to be able to reproduce the synaptic release variability observed in vitro. 
We began by implementing UVR at all synaptic contacts in the neocortical microcircuit model. As a result, our synaptic responses were highly variable in comparison against biological experiments. In order to study this problem, we recorded new 33 pairs of L5_TTPC strongly connected cells (Fig.1A up) and computed the amplitude and the CV of the amplitudes for each EPSP. In figure 1, we show two examples of experiments, one in vitro (Fig. 1A down red) and another in silico (Fig. 1B down blue). Differences in the shape and amplitude of the mean traces can be seen. The in vitro trace in red has a higher amplitude than the in silico in blue. Is also visible that the shape of the in silico mean trace is noisier than the in vitro, reflecting larger variability between protocol repetitions.

Although is possible to see differences between the amplitudes in the examples, the Kruskal-Wallis test of the distributions for the first EPSP amplitude (Fig. 1C) revealed no significant difference between the two data sets (mean \pm standard deviation EPSP values: $1.46 \pm 0.86 \mathrm{mV}$ for in vitro; 1.17 $\pm 0.57 \mathrm{mV}$ for in silico; $\mathrm{p}=0.15$ ) (significant if $\mathrm{p}<0.05$ ). However, the computation of the KruskalWallis test between the distributions of the CV for the first EPSP amplitude (Fig. 1E) revealed a significant difference between both data sets (mean CV values: $0.38 \pm 0.21$ for in vitro; $0.45 \pm 0.11$ for in silico; $\mathrm{p}=0.0092$ ). Consequently, computing the CV profile for the EPSP amplitudes for every stimulus showed a large difference between both data sets (Fig. 1D). The previous statistic test computed for the CV of the EPSP amplitudes showed very significant differences $\left(\mathrm{p}<10^{-9}\right)$ for the rest of the EPSPs. The distributions (Fig. 1C and E) were normalized to the respecting sample size such that the sum of products of width and height of each column is equal to the total count (33 for in vitro, 100 for in silico). Moreover, the cross validated Kolmogorov-Smirnov test for two-dimensional data (Press and Teukolsky, 1988) showed a significant difference between the first EPSP amplitude against the $\mathrm{CV}$ of the first EPSP amplitude for each data set $(\mathrm{p}=0.0022$; significant if $\mathrm{p}<0.2)$ (Fig. $1 \mathrm{~F})$ demonstrating that both data sets are different. As we can observe in figure $1 \mathrm{~F}$, in vitro data (red dots) are more dispersed than in silico (blue dots).

This evidence motivated us to implement the MVR hypothesis as it is known to provide the synapse with a larger range of variability, from too variable to almost no variance (Brémaud et al., 2007; Wang et al., 2006). 
A In Vitro
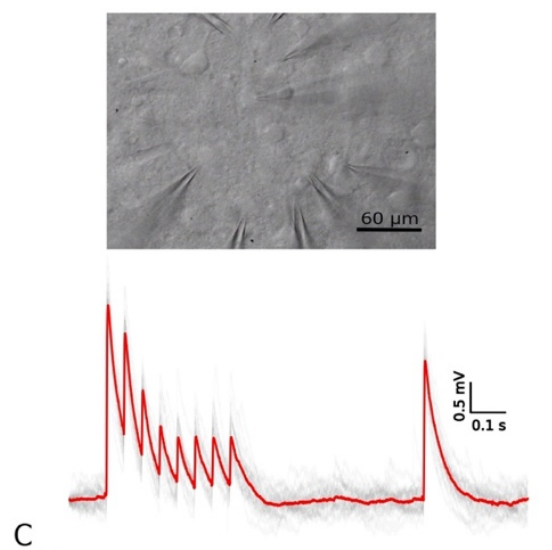

C
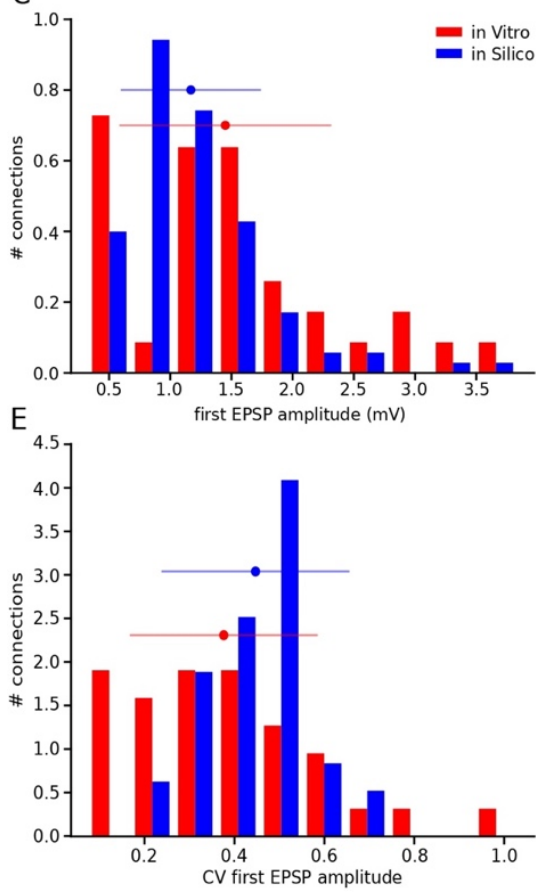

B In Silico
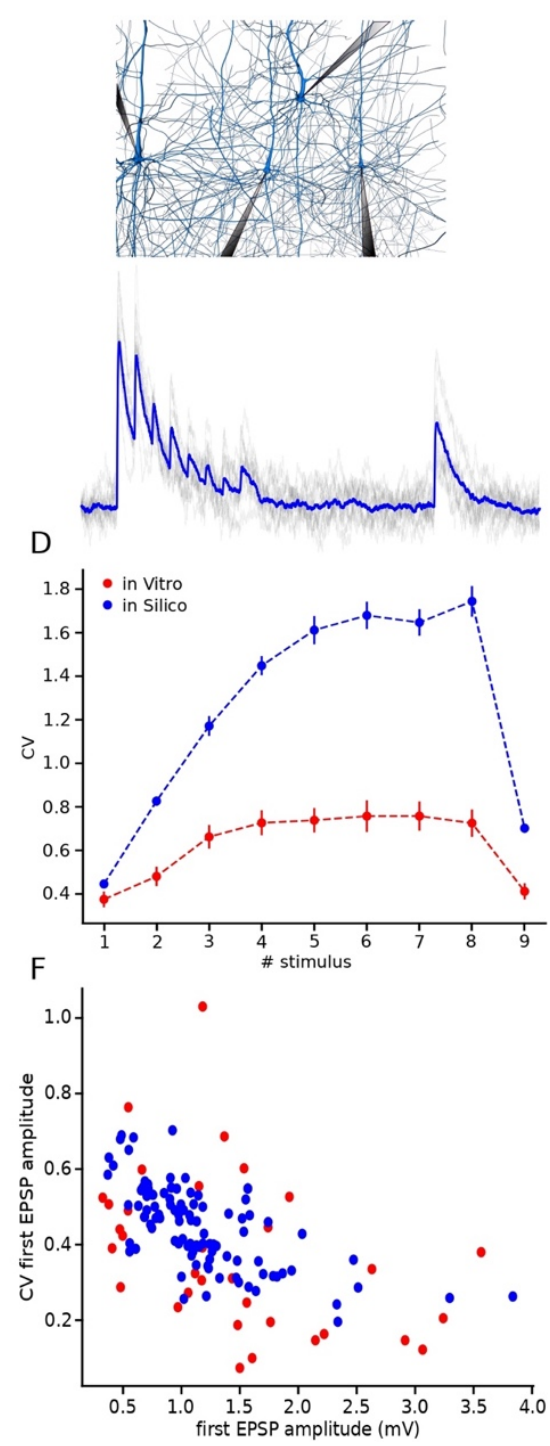

Figure 1. With the UVR hypothesis it was not possible to reproduce the variability observed in vitro. (A)(up) Picture of a multiple whole cell patch-clamp recording in L5_TTPC connections. (down) In vitro mean voltage trace (red) of 20 protocol repetitions (grey). (B)(up) Picture of a patch-clamp in silico experiment performed on L5_TTPC connections from the data-driven model of the rat cortex microcolumn. (down) In silico mean voltage trace (blue) of 20 protocol repetitions (grey). (C) Histogram showing the distribution of the first EPSP amplitude for in vitro (red) and for in silico (blue) experiments. (D) Mean CV profiles for the in vitro (red) and the in silico (blue) experiments. (E) CV distribution of the first EPSP amplitude for in vitro (red) and in silico (blue) data sets. (F) Raster plot of the first EPSP amplitude against the $\mathrm{CV}$ of the first EPSP amplitude for in vitro (red) and in silico (blue) experiments. In the distributions and the CV profile, dots represent the mean and vertical and horizontal bars represent the standard deviation of all the experiments respectively. 


\subsection{Extracting values for the TM-model and noise calibration}

To be able to compare the in vitro and the in silico experiments in a very precise manner, we had to extract certain parameters from the 33 in vitro traces mentioned before. The parameters in which we were interested were those related with the TM-model, to describe the synapse behavior, and the ones related with voltage fluctuations as the membrane noise.

The important synaptic parameters for the TM-model were extracted from the deconvolution of in vitro each averaged trace (Fig. 2B), so we were able to extract the values of the peaks from the same voltage level. We obtained three distributions, one per each parameter. For U we obtained a normal distribution with a mean value of $0.38 \pm 0.1$ (Fig. 2C), D fitted a gamma distribution with a mean value of $365.6 \pm$ $100.15 \mathrm{~ms}$ (Fig. 2D) and F was also fitted to a gamma distribution with mean $25.71 \pm 45.87 \mathrm{~ms}$ (Fig. 2E). These values were similar to the values found in previous studies (Tsodyks and Markram, 1997b; Wang et al., 2002). Later we implemented these parameters as the correspondent specific distributions into our model. The next step was to estimate the $g_{\max }$. To approximate it, we ran simulations with different $g_{\max }$, computed the amplitude of the first EPSP and compared this value with the values from the in vitro data set. We kept the $g_{\max }$ from which the correspondent EPSP amplitude was similar to the one found in the in vitro. The value we obtained was $1.54 \pm 1.20 \mathrm{nS}$, which is in accordance with values found in previous works (Markram et al., 1997). These parameters helped us to simulate the synaptic behavior of L5_TTPC connections. (Values expressed by mean \pm standard deviation).

A
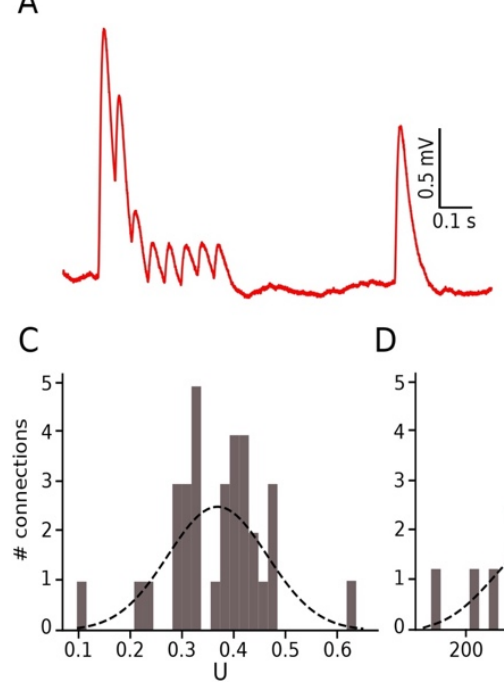

B
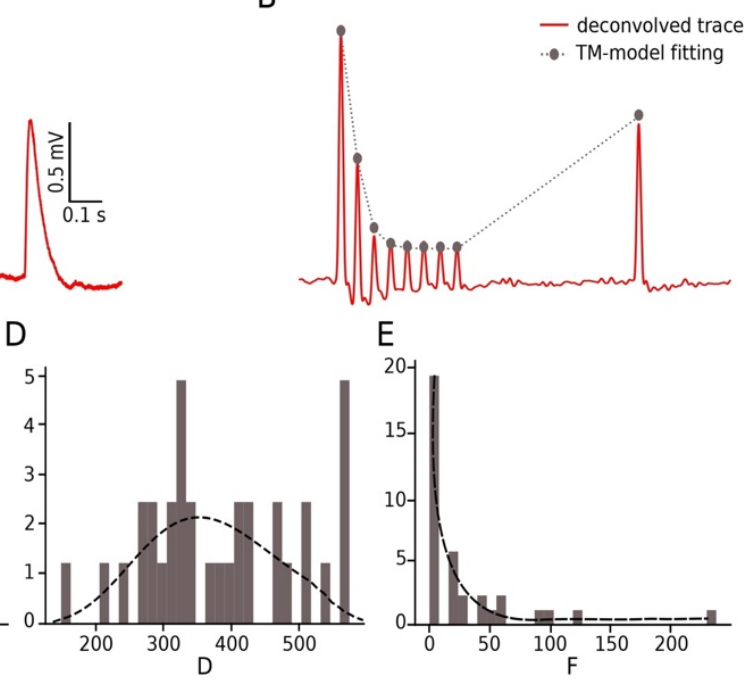

E

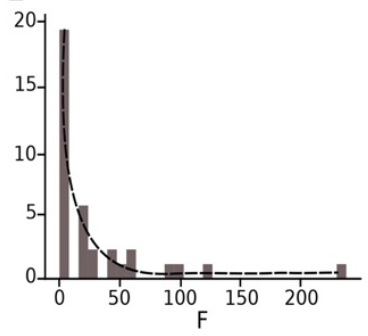

Figure 2. Fitting in vitro data to the TMmodel. (A) Example of an in vitro mean voltage trace of L5_TTPC connection. (B) Corresponding deconvolved voltage trace (red) with the fit to the deterministic TMmodel (grey). (C) Distribution of the probability of release parameter (U), (D) distribution of the time to recovery from depression (D) and (E) distribution of the time to recovery from facilitation $(\mathrm{F})$. Values obtained from the fitting to the TM-model of 33 in vitro connections. 
Other important parameters to be calibrated are the ones related with membrane noise. For this purpose, we implemented an OU-process, for which we needed to extract some parameters from each individual sweep of the in vitro traces. The values that we got from this calibration were $\sigma=0.22 \pm 0.10$ (Fig. 3B up) and $\tau=28.2 \pm 3.5$ (Fig. 3B down). After that we added synthetic voltage noise to each of the simulated sweeps generated each time as a new OU-process (Fig. 3C). With all these: the synaptic U, $\mathrm{D}, \mathrm{F}$ and $\mathrm{g}_{\max }$ and the membrane noise parameters, we captured the synaptic variability for this specific connection.
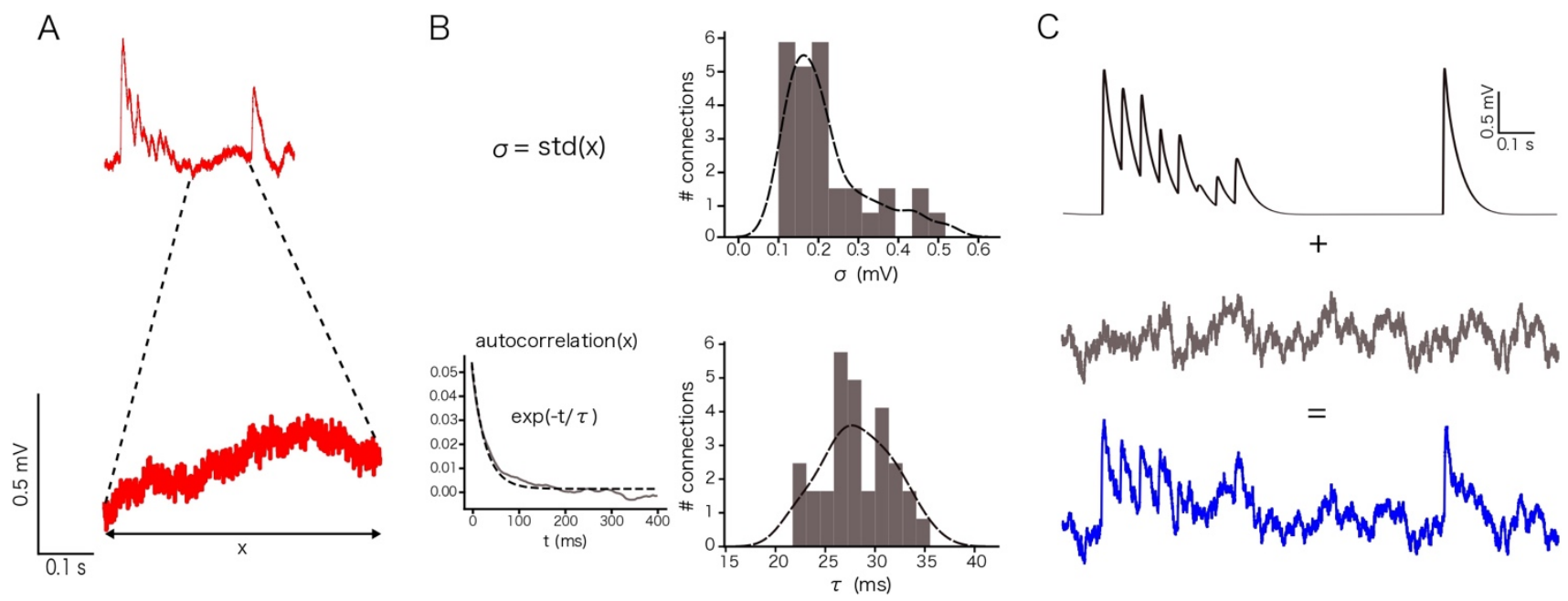

Figure 3. Noise calibration. (A) Example of an in vitro single protocol repetition (up). (down) Zoom over $400 \mathrm{~ms}$ segment used to compute the parameters for noise calibration. (B) Distribution of $\sigma$ (up) and $\tau$ (down). $\sigma$ was computed as the standard deviation of the voltage segment. $\tau$ was computed by fitting the voltage segment autocorrelation to an exponential. The distributions show the mean value $\mathrm{s}$ for the 33 in vitro connections. (C) (up) Single in silico trace without noise, (middle) OU-process generated to be added to the single in silico trace and (down) the noisy single protocol repetition that is the result of adding the previous two traces.

\subsection{Optimizing $\mathrm{N}_{\mathrm{RRP}}$ for L5_TTPC connections}

After defining all the needed parameters, we could run simulations with different $\mathrm{N}_{\text {RRPs }}$ with values between 1 to 14 (see methods 2.4) and compare them against the CV obtained from the in vitro data computed through the JKK approach. We could observe the relation between $\mathrm{N}_{\mathrm{RRP}}$ and the CV for this specific connection (Fig. 4C). As a first result we could observe that the CV for the first EPSP amplitude was higher when $\mathrm{N}_{\text {RRP }}$ was smaller. Therefore, for UVR like connections the variability between sweeps is larger than for MVR like connections. This result was in agreement with previous studies (Brémaud et al., 2007; Wang et al., 2006) and is also reflected on the in silico experiments run 
on the microcircuit with $\mathrm{N}_{\mathrm{RRP}}=1$ (Fig. 4B, up) and $\mathrm{N}_{\mathrm{RRP}}=20$ (Fig. 4B, down) used as examples to show how the variability and shape of the connections changes with the number of released vesicles.

In order to determine $\mathrm{N}_{\mathrm{RRP}}$, we computed the $\mathrm{CV}$ profiles of the in silico experiments performed with different $\mathrm{N}_{\mathrm{RRPs}}$ then by measuring the mean square distance (Fig. 4E) for each of them against the in vitro CV profile (Fig. 4D), we found that for this specific cell connection the minimum error was achieved by the simulation with $\mathrm{N}_{\mathrm{RRP}}=3.78 \pm 1.65$. This result showed us that MVR is a process that happens in the rat cortex between L5_TTPCs and is also in agreement with other studies (Loebel et al., 2009a; Rollenhagen et al., 2018).

A

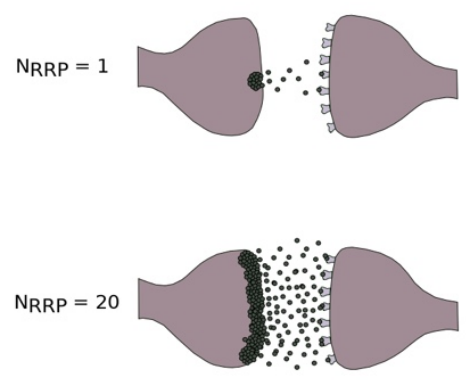

D

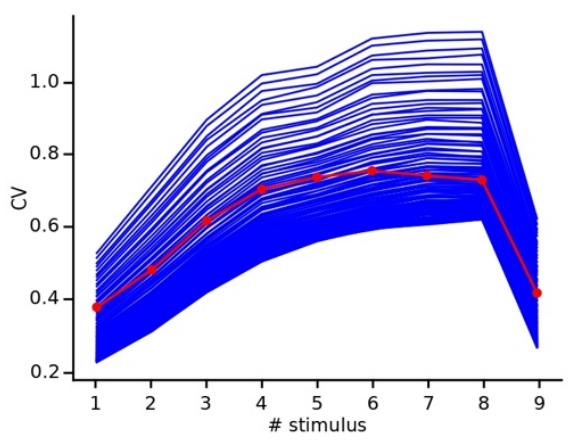

B

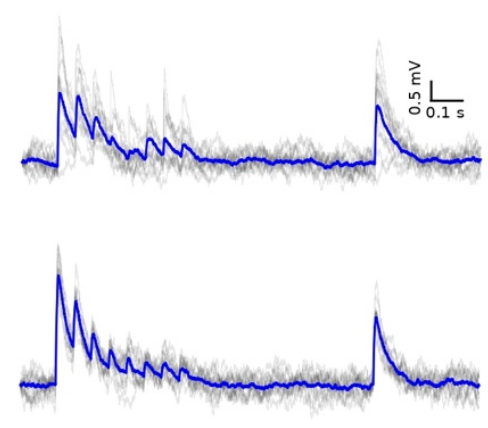

E

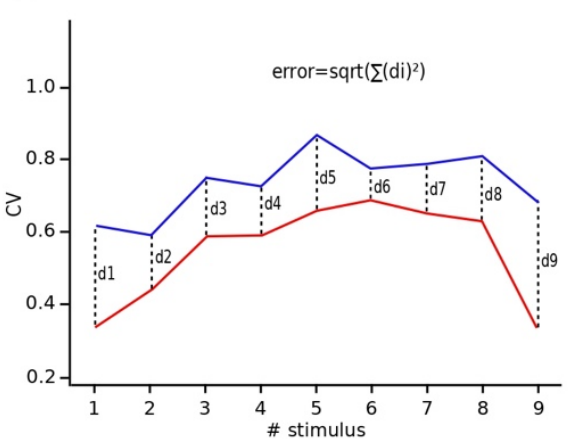

C

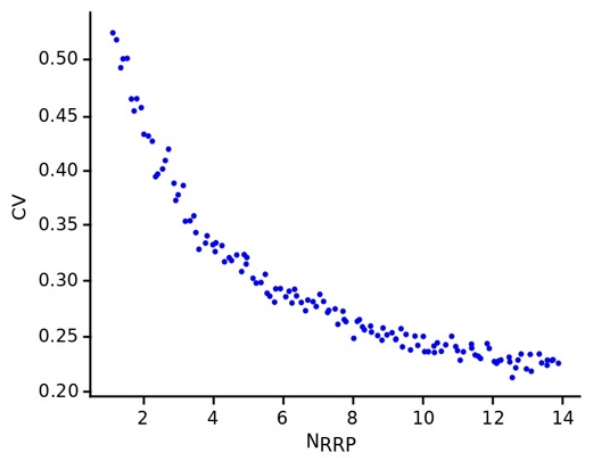

$\mathrm{F}$

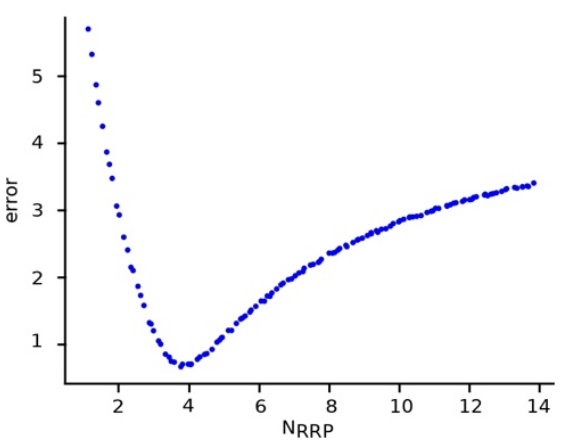

Figure 4. NRRP computation. (A) Illustration showing one synaptic connection releasing neuro transmitters from only one vesicle (up) and the same synaptic connection releasing neurotransmitters from twenty vesicles (down). (B) The corresponding effect of releasing neurotransmitters from one (up) or from twenty (down) vesicles reflected on the variability and shape of the in silico traces. The mean voltage traces are painted in blue while each protocol repetition is represented in grey. (C) Diagram showing the effect of $\mathrm{N}_{\mathrm{RRP}}$ over the CV. (D) Mean CV profile for the in vitro (red) and all the in silico connections with different $\mathrm{N}_{\text {RRP }}$ values. (E) Diagram explaining the mean square distance computation. (F) $\mathrm{N}_{\text {RRP }}$ against error, showed a clear minimum around the value obtained for this specific connection. 


\subsection{Implementing MVR improved the variability of the synapses in the model}

Once we obtained the value for the $\mathrm{N}_{R R P}$ parameter we wanted to compare the previous result in which only one vesicle was released (Fig. 1) and observe if the implementation of the MVR hypothesis really improved the model behavior, showing a result closer to the biological data. For this reason, we computed the distributions for the first EPSP amplitude, the CV of the first EPSP amplitude and the CV profile of the EPSP amplitudes for all the pulses. We could observe that the shape and the amplitude of the in silico trace (Fig. 5B) is similar to the in vitro trace (Fig. 5A), in contrast to the comparison with the UVR like in silico trace (Fig 1B, down). The CV profile for all the EPSPs (Fig. 5D) for in silico (blue) is also closer to the in vitro (red) than the one computed applying UVR (Fig. 1D). Although our model has a slightly higher $\mathrm{CV}$ for the $6^{\text {th }}, 7^{\text {th }}$ and $8^{\text {th }}$ EPSPs, the Kruskal-Wallis test showed no significant differences between both CV profiles for any of the EPSPs, demonstrating that the MVR hypothesis improved the synaptic variability of the circuit.

A In Vitro
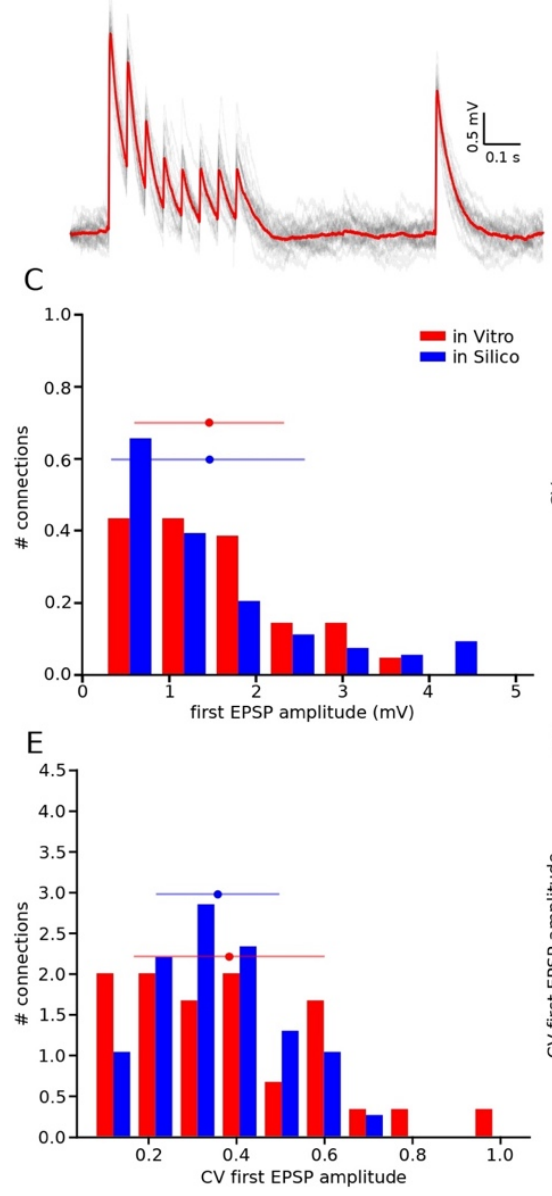

B In Silico
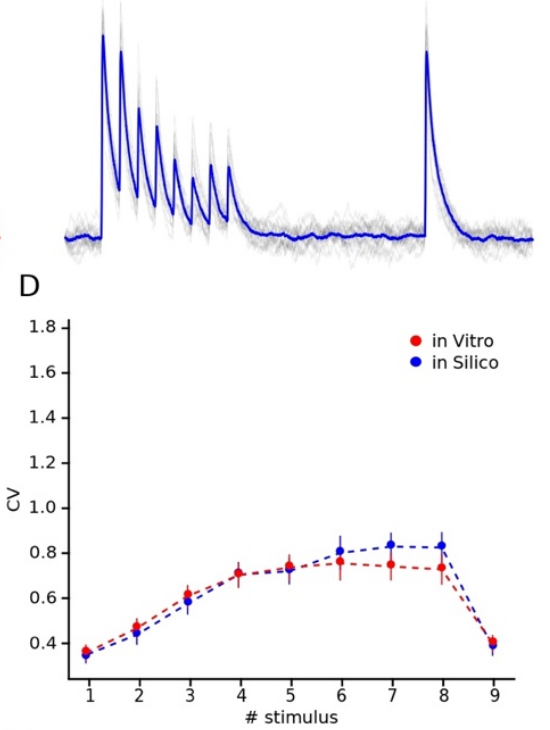

$\mathrm{F}$

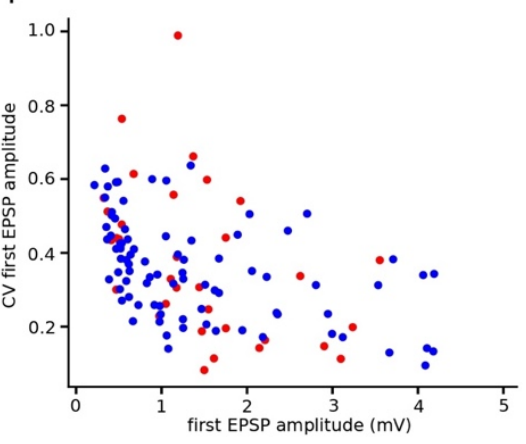

Figure 5. Releasing multiple vesicles improved the variability of the model.

(A) In vitro mean voltage trace (red) of 20 protocol repetitions (grey) (same as in figure 1A down). (B) In silico mean voltage trace (blue) of 20 protocol repetitions (grey). (C) Distribution of the first EPSP amplitude for in vitro (red) and for the in silico (blue) experiments. (D) Mean CV profiles for the in vitro (red) and the in silico (blue) experiments. (E) CV Distribution of the first EPSP amplitude for in vitro (red) and the in silico (blue) data sets. (F) Raster plot of the first EPSP amplitude against the CV of the first EPSP amplitude for in vitro (red) and in silico (blue) experiments. All the in silico experiments are done with the $\mathrm{N}_{\mathrm{RRP}}$ value that produces the minimum error. In the distributions and the $\mathrm{CV}$ profile, dots represent the mean and vertical and horizontal bars represent the standard deviation of all the experiments. 
Another result that supported the improvement, was shown on the distributions for the first EPSP amplitude (Fig. 5C) and for the CV of the first EPSP amplitude (Fig. 5E). Both had a mean value closer than the one from the UVR like model as the statistic test showed no significant difference (mean EPSP values: $1.46 \pm 0.86 \mathrm{mV}$ for in vitro; $1.46 \pm 0.95 \mathrm{mV}$ for in silico; $\mathrm{p}=0.69)$ (mean CV values: $0.38 \pm$ 0.21 for in vitro; $0.35 \pm 0.13$ for in silico; $\mathrm{p}=0.86$ ). The distributions (Fig. $5 \mathrm{C}$ and $\mathrm{E}$ ) were normalized to the respecting sample size such that the sum of products of width and height of each column is equal to the total count (33 for in vitro, 85 for in silico). Furthermore, the cross validated KolmogorovSmirnov test showed no significant difference between the first EPSP amplitude against the CV of the first EPSP amplitude for each data set $(\mathrm{p}=0.29)(\mathrm{Fig} .5 \mathrm{~F})$, meaning that both data sets could come from the same population.

\section{5 $\quad \mathrm{N}_{\mathrm{RRP}}$ prediction for other cell connections}

Moreover, we extended this method to other cell connections for which we have models in our circuit, but no raw in vitro data sets. In this occasion we collected CVs data from the literature all of them found only for the $1^{\text {st }}$ EPSP amplitude.

Before computing the $\mathrm{CV}$ for the new cell connections, we had to consider that these $\mathrm{CV}$ were not computed using the JKK bootstrapping approach. From our previous analysis we know that the CV computed through the JKK method has a slightly different value than the CV computed without this mathematical approach. In the case of L5_TTPC connections in vitro data set the CV $=0.31 \pm 0.14$ while the $\mathrm{CV}_{\mathrm{JKK}}=0.38 \pm 0.21$ (CVs computed only for the first pulse), but the $\mathrm{N}_{\mathrm{RRPs}}$ computed after 50 iterations in both cases were mostly the same with no significant difference between them (no-JKK $\mathrm{N}_{R R P}=2.41 \pm 1.08$ and $J K K N_{R R P}=2.73 \pm 1.22 ; p<0.01$ ) (Fig. 6A and B respectively). This value is smaller than the previous $\mathrm{N}_{\mathrm{RRP}}$ obtained from comparing the $\mathrm{CV}$ for all the pulses, but it was expected as in the previous analysis we didn't reach the exact $\mathrm{CV}$ value for the $1^{\text {st }}$ pulse, although there was no significant difference.

As we knew that the JKK bootstrapping method provide us with a more accurate manner for computing the EPSP amplitudes, we applied a transformation from CV to $\mathrm{CV}_{\mathrm{JKK}}$ (Fig. 6C). We illustrate this transformation in figure 6 with L5_TTPC. First, we computed the CV of the first EPSP amplitude without (Fig. 6A) and with the JKK (Fig. 6B) analysis. Second, we represented both CVs in the same

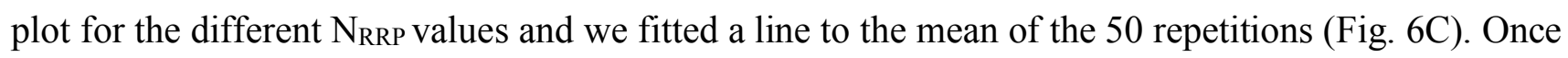


we obtained the linear equation we were able to determine the correspondent CV value computed with the JKK approach (Fig. 6B) for this connection we obtained $\mathrm{CV}_{\mathrm{JKK}}=0.39 \pm 0.15$ with a correspondent $\mathrm{N}_{R R P}=2.84 \pm 1.34$. We did that for every connection for which we could find data in the literature and our simulation matched the variability (Table 1).

\begin{tabular}{|c|c|c|c|}
\hline Connection type & Literature data & $\begin{array}{l}\text { Jack-Knife } \\
\text { conversion }\end{array}$ & Prediction \\
\hline & $\mathrm{CV}$ & $\mathrm{CV}$ & $\mathrm{N}_{\mathrm{RRP}}$ \\
\hline L23_NBC_LBC-L23_PC & $\begin{array}{l}0.40 \pm 0.09 \\
\text { (Wang, 2002) }\end{array}$ & $0.38 \pm 0.21$ & $1.96 \pm 0.98$ \\
\hline L23_PC-L23_PC & $\begin{array}{c}0.33 \pm 0.18 \\
\text { (Feldmeyer, 2006) }\end{array}$ & $0.48 \pm 0.23$ & $2.60 \pm 1.28$ \\
\hline L4_SSC-L23_PC & $\begin{array}{c}0.27 \pm 0.13 \\
\text { (Feldmeyer, 2002) }\end{array}$ & $0.37 \pm 0.09$ & $1.81 \pm 0.37$ \\
\hline L4_SSC-L5_TPC:C & $\begin{array}{c}0.33 \pm 0.20 \\
\text { (Feldemyer, 2005) }\end{array}$ & $0.46 \pm 0.15$ & $1.26 \pm 0.50$ \\
\hline L5_TTPC-L5_SBC & $\begin{array}{l}0.32 \pm 0.08 \\
\text { (Wang, 2002) }\end{array}$ & $0.34 \pm 0.16$ & $1.82 \pm 0.90$ \\
\hline L5_TTPC-L5_TTPC & $\begin{array}{c}0.31 \pm 0.14 \\
\text { (Our value) }\end{array}$ & $0.39 \pm 0.15$ & $2.84 \pm 1.34$ \\
\hline
\end{tabular}

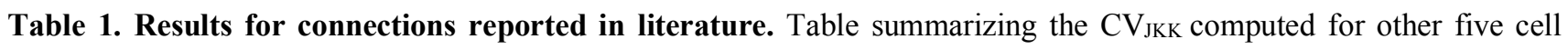
connections through the collection of data from literature and applying the JKK conversion explained in figure 6. For the L5_TTPC connection we used the CV computed in this work from our in vitro data set. L23_NBC_LBC: layer 2 and 3 nest and large basket cells; L23_PC: pyramidal cells in layer 2 and 3; L4_SSC: layer 4 spiny stellate cells; L5_TPC:C: thick tuft pyramidal cells that receive projections from thalamus; L5_SBC: small basket cells from layer 5.

The results are summarized in Table 1 where we can observe that we were able to generalize for other five neuronal connections of the rat neocortex. We found that for layer 4 spiny stellate (L4_SSC) to layer 5 pyramidal cell connections that receive thalamic projections (L5_TPC:C) the transmission is mostly UV $\left(\mathrm{N}_{\mathrm{RRP}}=1.26 \pm 0.50\right)$, while for the rest of the connections in the table the average number of vesicles ready to be released at the presynaptic terminal is between 2 to $3\left(\mathrm{~N}_{\mathrm{RRP}}=2.60 \pm 1.28\right.$ for L23_PC-L23_PC; NRRP $=1.96 \pm 0.98$ for L23_NBC_LBC-L23_PC; NRRP $=1.81 \pm 0.37$ for L4_SSCL23_PC and NRRP $=1.82 \pm 0.90$ for L5_TTPC-L5_SBC). 

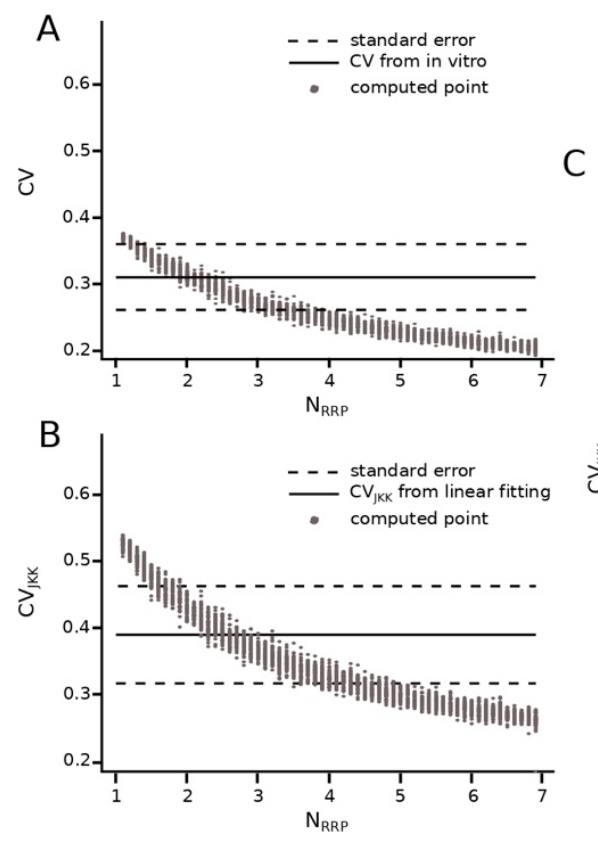

C

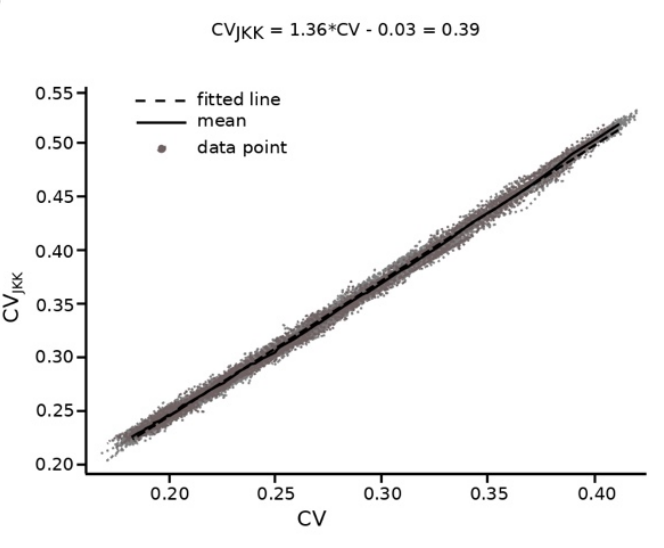

Figure 6. Extension of the method for connections reported in literature. Transformation from $\mathrm{CV}$ to $\mathrm{CV}$ JKK using L5_TTPC connection as example (A) CV computed for different NRRP. Solid black line represents the CV computed for

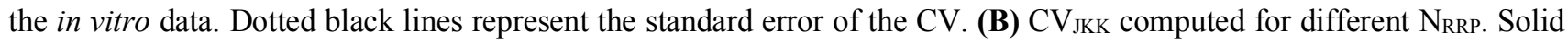
black line represents the $\mathrm{CV}_{\text {JKK }}$ obtained from the lineal fitting on $\mathbf{C}$. Dotted black lines represent the standard error for this $\mathrm{CV}_{\text {JKK. }}$ (C) $\mathrm{CV}$ to $\mathrm{CV}_{\text {JKK }}$ transformation. Solid black line represents the mean of the 50 iterations and dotted black line represent the linear fitting which equation is at the top of the plot. In $\mathbf{A}$ and $\mathbf{B}$ the grey dots show the 50 iterations from which we extract the best $\mathrm{N}_{\mathrm{RRP}}$ as the one corresponding with the closest $\mathrm{CV}$.

These results confirmed that implementing MVR in the model improved the synaptic dynamics with regard to the biological data. We found that for L5_TTPC connections $\mathrm{N}_{\mathrm{RRP}}$ has variable value between 3 and 4 per synaptic contact. Furthermore, we predicted that MVR is most likely to happen in other cortical cell connections, supporting the idea that multiple vesicles are released in the neocortex influencing synaptic variability and information transmission.

\section{Discussion}

In this work we computed the $\mathrm{N}_{\text {RRP }}$ based on the previous work of Loebel and colleagues of 2009 (Loebel et al., 2009a) but we extended it to every active connection in a synapse. This approach is based on the comparison of the EPSPs amplitudes CV between a set of in vitro and in silico experiments with different $\mathrm{N}_{\mathrm{RRP}}$ values performed in a data driven model of the rat somatosensory cortex (Markram et al., 2015) for a specific neuronal connection. The CV of the amplitude distributions is a currently 
used marker of the concentration of neurotransmitter in the synaptic cleft and for the postsynaptic receptor occupancy (Auger and Marty, 2000; Faber and Korn, 1991). For example, if a big quantity of neurotransmitter is released into the synaptic cleft then a high amplitude EPSP would be generated into the postsynaptic terminal. However, a large fraction of receptors would be occupied as well and consequently it would be more difficult to generate a second EPSP if more neurotransmitter is released. Thus, it is possible to measure the variability of the amplitude considering that high variability represents a small number of released vesicles.

\subsection{UVR could not reproduce the variability observed into the biological data}

Through this analysis we found that the UVR hypothesis did not reproduce the variability observed on the in vitro traces, in fact the $\mathrm{CV}$ profile for the in silico experiments was significantly larger, although the first EPSP amplitude was not statistically different. This result suggested us that the MVR hypothesis could be the appropriate approach to be considered for our model. On one hand, this idea differed from previous studies (Gulyás et al., 1993; Murphy et al., 2004; Redman, 1990) in which they claim that from each active contact in a synapse only one vesicle as most could be released, suggesting that the biological variability may come from changes in the quantal size. On the other hand, there were newer research studies that supported our idea of implementing the MVR hypothesis to improve our model (Brémaud et al., 2007; Hardingham et al., 2010; Huang et al., 2010; Loebel et al., 2009a; Rudolph et al., 2015). This discrepancy between works could come because they were studying different brain regions, with different experimental protocols, different species or different cell connections.

Before comparing both data collections, we extracted some important parameters from the in vitro group of data. First, we computed the parameters related with the deterministic model for short-term synaptic depression. For this purpose, we had to select only the depressing connections which $1^{\text {st }}$ EPSP amplitude was within the range of the in vitro data set and apply the deconvolution approach in order to be able to fit the peaks of each trace in the most precise possible manner. The values obtained were similar to values found in previous researches (Tsodyks and Markram, 1997b; Wang et al., 2006). Second, we calibrated the synaptic noise which represented the trial-to-trial variability into the synapse. Many studies support the idea that background synaptic noise is not only noise, but an addition of various meaningful procedures (Azouz and Gray, 1999; Faisal et al., 2008). Synaptic noise is classically shown in the spontaneous miniature postsynaptic currents, which is thought to be the result of spontaneous vesicle released (Fatt and Katz, 1950). This noise can influence not only the synaptic 
variability but also the transmission of information (Jacobson et al., 2005). Thus, while some studies did not support our idea of the contribution of the number of vesicles in the synaptic noise (Mackenzie et al., 2000), many others (Faisal et al., 2008; Franks et al., 2003; Pulido and Marty, 2017) encouraged us to complement our model with noise calibration.

\subsection{L5_TTPC synapses are driven by multiple vesicles}

In this research increasing the $\mathrm{N}_{\mathrm{RRP}}$ improved the variability of the model, yielding synapses that behaved more similarly to the ones found in the biological system. Consequently, for cell connections between L5_TTPCs studied with a stimulation train at $20 \mathrm{~Hz}$ the resultant $\mathrm{N}_{\mathrm{RRP}}$ mean was $3.78 \pm 1.65$ within a range between 1 to 9 vesicles. Considering that the number of active contacts found in L5_TTPC connections is between 4 to 8 (Markram et al., 1997), our prediction was that the range of total number of release sites for a synapse would be between 4 to 72 . This estimation is in the range of previous studies in which they found that the range of the docked vesicles in L5_TTPC synapses was 2 - 30 (Rollenhagen and Lübke, 2006). Our predicted range is also consistent with previous estimates (Loebel et al., 2009a)(7 - 170), although in this case our range is considerably smaller. This difference could be due to the different approaches for the simulation: with the Montecarlo approach in the case of Loebel or with the data-driven model of the microcircuit in our case. Our result is also in accordance with a recent study in which they found that in L5B pyramidal cells of the rat somatosensory cortex at individual synaptic contacts the number of vesicles ready to be released ranged between 1.2 to 12.8 (Rollenhagen et al., 2018), although their mean value is slightly larger (5.40 \pm 1.24$)$. This difference could be due to the fact that we are considering all the TTPC in Layer 5 and not only the neurons in Layer 5B. Evidence from the mouse neuromuscular junction indicates a huge number of vesicles per active zone ( 1700) (Ruiz et al., 2011). This large difference could be due to the stimulation frequency, which was $100 \mathrm{~Hz}$ comparing with our stimulation at $20 \mathrm{~Hz}$, but it could be also due to the functionality. Synapses in the cortex probably need more variability as these areas are known to process information, while synapses between motoneurons and muscular fibers should be more reliable. Probably, pointing out the importance of MVR not only for the transmission of the information, but also as an important factor for defining the synaptic functionality.

The CV computed from the in silico experiments was slightly different without signification for the $6^{\text {th }}, 7^{\text {th }}$ and $8^{\text {th }}$ EPSPs. We also found 1 vesicle of difference between the comparison with the train or comparing only the first pulse. It is known that many mechanisms are involved in vesicular release as 
the membrane fusion, receptor saturation, vesicle recycling or the barrier effect of the glia (Rizo and $\mathrm{Xu}, 2015$; Rudolph et al., 2015; Stevens, 2003). We should take in consideration all these complex mechanisms for the future and complete our cortical model with them.

\subsection{MVR also occurs in other cell connections of the rat neocortex}

We extended the method to connections involving other cell types in the neocortex for which we had models and data from literature. We succeeded on five connections from which we concluded that on average the number of vesicles ready to be released is between 2 and 3. Although some studies did not support our result (Silver et al., 2003), many others support the idea of MVR as a process that happens in the neocortex (Zhang and Peskin, 2015) and actually that not necessarily the number of vesicles does not necessarily have to be the same, but that it could change from one layer to another (Brémaud et al., 2007). Other studies agreed with our result, suggesting that we achieve this result because the data that we were comparing with come from the somatosensory cortex (Huang et al., 2010). Consequently, it might be possible that MVR happens in some cortical areas but not in others, demonstrating that vesicle release is relevant for signal processing.

We had to extrapolate some data, for L5_TPC-L5_SBC we used the data from the work of Wang and colleagues of 2002 in which they describe the anatomy and synaptic behavior of basket interneurons in layers $2-4$ (Wang et al., 2002). We took that decision since we could not find any reference from literature studying the necessary aspects of the synapse between pyramidal and basket cells in layer 5 . If in the future data becomes available, it would be possible that the $\mathrm{N}_{R R P}$ for this connection changes. Another problem was that we did not manage to generalize all the connections for which we have models because we did not find any data in the literature or because the experimental data from the literature provided CV distributions with some very weak connections. In our model, even though we have weak connections, the $\mathrm{CV}$ distribution has a lower mean because most of the connections are stronger than those found in previous researches. Some studies provide a functional view of networks formed by weak connections as a manner to keep synchronicity in the brain (Bruno and Sakmann, 2006; Ren et al., 2017), showing the importance of the weak connections. We should study in depth this issue and come up with a solution in order to unravel the $\mathrm{N}_{\mathrm{RRP}}$ for the rest of the connections.

In this work we provide a method to computed the $\mathrm{N}_{\text {RRP }}$ per active contact in a synapse. This method is an extension of Loebel and colleague's work from 2009. Through the comparison between a set of in vitro and in silico data of the EPSP amplitude CV, we were able to find the correspondent $\mathrm{N}_{\text {RRP. The }}$ 
results obtained by this method helped us to understand that MVR is a process that very likely happens in some cortical areas of the rat brain and that can be an important mechanism for the correct functioning of the information transmission through the brain.

\section{$5 \quad$ Conflict of Interest}

The authors declare that the research was conducted in the absence of any commercial or financial relationships that could be construed as a potential conflict of interest.

\section{Author Contributions}

NB-Z developed and performed the data analysis and the in silico experiments, wrote the manuscript and illustrated the figures. JR developed and performed some data analysis. GC developed and performed some data analysis and some in silico experiments. RP designed and performed the in vitro experiments. HM critical revision of the manuscript. SR contributed to the writing and critical revision of the article. EM contributed to data interpretation, writing and critical revision of the manuscript.

\section{$7 \quad$ Funding}

The ETH Domain for the Blue Brain Project (BBP); The Human Brain Project through the European Union Seventh Framework Program (FP7/2007-2013) under grant agreement no. 604102 (HBP) and from the European Union H2020 FET program through grant agreement no. 720270 (HBP SGA1); The Cajal Blue Brain Project (MINECO); The BlueBrain 4 BlueGene/Q and BlueBrain 5 system are financed by ETH Board Funding to the Blue Brain Project as a National Research Infrastructure and hosted at the Swiss National Supercomputing Center (CSCS).

\section{$8 \quad$ Reference}

Auger, C., and Marty, A. (2000). Quantal currents at single-site central synapses. J. Physiol. 526 Pt 1, $3-11$.

Auger, C., Kondo, S., and Marty, A. (1998). Multivesicular release at single functional synaptic sites in cerebellar stellate and basket cells. J. Neurosci. Off. J. Soc. Neurosci. 18, 4532-4547.

Azouz, R., and Gray, C.M. (1999). Cellular mechanisms contributing to response variability of cortical neurons in vivo. J. Neurosci. Off. J. Soc. Neurosci. 19, 2209-2223.

Betz, W.J. (1970). Depression of transmitter release at the neuromuscular junction of the frog. J. Physiol. 206, 629-644. 
Biró, A.A., Holderith, N.B., and Nusser, Z. (2005). Quantal size is independent of the release probability at hippocampal excitatory synapses. J. Neurosci. Off. J. Soc. Neurosci. 25, 223-232.

Boucher, J., Kröger, H., and Sík, A. (2010). Realistic modelling of receptor activation in hippocampal excitatory synapses: analysis of multivesicular release, release location, temperature and synaptic cross-talk. Brain Struct. Funct. 215, 49-65.

Bradley Efron, and Robert J. Tibshirani (1994). An introduction to the bootstrap.

Brémaud, A., West, D.C., and Thomson, A.M. (2007). Binomial parameters differ across neocortical layers and with different classes of connections in adult rat and cat neocortex. Proc. Natl. Acad. Sci. U. S. A. 104, 14134-14139.

Bruno, R.M., and Sakmann, B. (2006). Cortex is driven by weak but synchronously active thalamocortical synapses. Science $312,1622-1627$.

Christie, J.M., and Jahr, C.E. (2006). Multivesicular release at Schaffer collateral-CA1 hippocampal synapses. J. Neurosci. Off. J. Soc. Neurosci. 26, 210-216.

Conti, R., and Lisman, J. (2003). The high variance of AMPA receptor- and NMDA receptormediated responses at single hippocampal synapses: evidence for multiquantal release. Proc. Natl. Acad. Sci. U. S. A. 100, 4885-4890.

Del Castillo, J., and Katz, B. (1954). Quantal components of the end-plate potential. J. Physiol. 124, 560-573.

Enrico Bibbona (2008). The Ornstein-Uhlenbeck process as a model of a low-pass filtered white noise. Metrologia 45, S117-S126.

Faber, D.S., and Korn, H. (1991). Applicability of the coefficient of variation method for analyzing synaptic plasticity. Biophys. J. 60, 1288-1294.

Faisal, A.A., Selen, L.P.J., and Wolpert, D.M. (2008). Noise in the nervous system. Nat. Rev. Neurosci. 9, 292-303.

Fatt, P., and Katz, B. (1950). Some observations on biological noise. Nature 166, 597-598.

Franks, K.M., Stevens, C.F., and Sejnowski, T.J. (2003). Independent sources of quantal variability at single glutamatergic synapses. J. Neurosci. Off. J. Soc. Neurosci. 23, 3186-3195.

Fuhrmann, G., Segev, I., Markram, H., and Tsodyks, M. (2002). Coding of Temporal Information by Activity-Dependent Synapses. J. Neurophysiol. 87, 140-148.

Fuhrmann, C.A., Segev I, T.M., and Christian Stricker (2004). Multiple mechanisms govern the dynamics of depression at neocortical synapses of young rats. J. Physiol. 557, 415-438.

Gordon, G.R.J. (2005). Noradrenaline Triggers Multivesicular Release at Glutamatergic Synapses in the Hypothalamus. J. Neurosci. 25, 11385-11395.

Gulyás, A.I., Miles, R., Sík, A., Tóth, K., Tamamaki, N., and Freund, T.F. (1993). Hippocampal pyramidal cells excite inhibitory neurons through a single release site. Nature 366, 683-687. 
Gupta, A., Wang, Y., and Markram, H. (2000). Organizing principles for a diversity of GABAergic interneurons and synapses in the neocortex. Science 287, 273-278.

Hardingham, N.R., Read, J.C.A., Trevelyan, A.J., Nelson, J.C., Jack, J.J.B., and Bannister, N.J. (2010). Quantal Analysis Reveals a Functional Correlation between Presynaptic and Postsynaptic Efficacy in Excitatory Connections from Rat Neocortex. J. Neurosci. 30, 1441-1451.

Hennig, M.H. (2013). Theoretical models of synaptic short term plasticity. Front. Comput. Neurosci. 7.

Huang, C.-H., Bao, J., and Sakaba, T. (2010). Multivesicular release differentiates the reliability of synaptic transmission between the visual cortex and the somatosensory cortex. J. Neurosci. Off. J. Soc. Neurosci. 30, 11994-12004.

Jacobson, G.A., Diba, K., Yaron-Jakoubovitch, A., Oz, Y., Koch, C., Segev, I., and Yarom, Y. (2005). Subthreshold voltage noise of rat neocortical pyramidal neurones. J. Physiol. 564, 145-160.

Korn, H., Triller, A., Mallet, A., and Faber, D.S. (1981). Fluctuating responses at a central synapse: $n$ of binomial fit predicts number of stained presynaptic boutons. Science 213, 898-901.

Korn, H., Bausela, F., Charpier, S., and Faber, D.S. (1993). Synaptic noise and multiquantal release at dendritic synapses. J. Neurophysiol. 70, 1249-1254.

Korn, H., Sur, C., Charpier, S., Legendre, P., and Faber, D.S. (1994). The one-vesicle hypothesis and multivesicular release. Adv. Second Messenger Phosphoprotein Res. 29, 301-322.

Liley, A.W., and North, K.A. (1953). An electrical investigation of effects of repetitive stimulation on mammalian neuromuscular junction. J. Neurophysiol. 16, 509-527.

Loebel, A., Silberberg, G., Helbig, D., Markram, H., Tsodyks, M., and Richardson, M.J.E. (2009). Multiquantal release underlies the distribution of synaptic efficacies in the neocortex. Front. Comput. Neurosci. 3, 27.

Maass, W., and Markram, H. (2002). Synapses as dynamic memory buffers. Neural Netw. Off. J. Int. Neural Netw. Soc. 15, 155-161.

Mackenzie, P.J., Kenner, G.S., Prange, O., and Murphy, T.H. (2000). Vesicle number does not predict postsynaptic measures of miniature synaptic activity frequency in cultured cortical neurons. Neuroscience 98, 1-7.

Markram, H., Lübke, J., Frotscher, M., Roth, A., and Sakmann, B. (1997). Physiology and anatomy of synaptic connections between thick tufted pyramidal neurones in the developing rat neocortex. J. Physiol. 500 ( Pt 2), 409-440.

Markram, H., Wang, Y., and Tsodyks, M. (1998). Differential signaling via the same axon of neocortical pyramidal neurons. Proc. Natl. Acad. Sci. U. S. A. 95, 5323-5328.

Markram, H., Muller, E., Ramaswamy, S., Reimann, M.W., Sanchez, C., Ailamaki, A., AlonsoNanclares, L., Antille, N., Arsever, S., and Kahou, G. (2015). Reconstruction and Simulation of Neocortical Microcircuitry. Cell. 
Mason, A., Nicoll, A., and Stratford, K. (1991). Synaptic transmission between individual pyramidal neurons of the rat visual cortex in vitro. J. Neurosci. Off. J. Soc. Neurosci. 11, 72-84.

Molnár, G., Rózsa, M., Baka, J., Holderith, N., Barzó, P., Nusser, Z., and Tamás, G. (2016). Human pyramidal to interneuron synapses are mediated by multi-vesicular release and multiple docked vesicles. ELife 5.

Murphy, G.J., Glickfeld, L.L., Balsen, Z., and Isaacson, J.S. (2004). Sensory neuron signaling to the brain: properties of transmitter release from olfactory nerve terminals. J. Neurosci. Off. J. Soc. Neurosci. 24, 3023-3030.

Nadkarni, S., Bartol, T.M., Sejnowski, T.J., and Levine, H. (2010). Modelling vesicular release at hippocampal synapses. PLoS Comput. Biol. 6, e1000983.

Neishabouri, A., and Faisal, A.A. (2014). Axonal noise as a source of synaptic variability. PLoS Comput. Biol. 10, e1003615.

Press, W.H., and Teukolsky, S.A. (1988). Kolmogorov-Smirnov Test for Two-Dimensional Data. Comput. Phys. 2, 74.

Pulido, C., and Marty, A. (2017). Quantal Fluctuations in Central Mammalian Synapses: Functional Role of Vesicular Docking Sites. Physiol. Rev. 97, 1403-1430.

Redman, S. (1990). Quantal analysis of synaptic potentials in neurons of the central nervous system. Physiol. Rev. 70, 165-198.

Ren, H.-P., Bai, C., Baptista, M.S., and Grebogi, C. (2017). Weak connections form an infinite number of patterns in the brain. Sci. Rep. 7, 46472.

Rizo, J., and Xu, J. (2015). The Synaptic Vesicle Release Machinery. Annu. Rev. Biophys. 44, 339367.

Rollenhagen, A., and Lübke, J.H.R. (2006). The morphology of excitatory central synapses: from structure to function. Cell Tissue Res. 326, 221-237.

Rollenhagen, A., Ohana, O., Sätzler, K., Hilgetag, C.C., Kuhl, D., and Lübke, J.H.R. (2018). Structural Properties of Synaptic Transmission and Temporal Dynamics at Excitatory Layer 5B Synapses in the Adult Rat Somatosensory Cortex. Front. Synaptic Neurosci. 10, 24.

Rudolph, S., Tsai, M.-C., von Gersdorff, H., and Wadiche, J.I. (2015). The ubiquitous nature of multivesicular release. Trends Neurosci. 38, 428-438.

Ruiz, R., Cano, R., Casañas, J.J., Gaffield, M.A., Betz, W.J., and Tabares, L. (2011). Active zones and the readily releasable pool of synaptic vesicles at the neuromuscular junction of the mouse. $\mathrm{J}$. Neurosci. Off. J. Soc. Neurosci. 31, 2000-2008.

Silver, R.A., Lubke, J., Sakmann, B., and Feldmeyer, D. (2003). High-probability uniquantal transmission at excitatory synapses in barrel cortex. Science 302, 1981-1984.

Stevens, C.F. (2003). Neurotransmitter release at central synapses. Neuron 40, 381-388. 
Südhof, T.C. (2000). The synaptic vesicle cycle revisited. Neuron 28, 317-320.

Tang, C.M., Margulis, M., Shi, Q.Y., and Fielding, A. (1994). Saturation of postsynaptic glutamate receptors after quantal release of transmitter. Neuron 13, 1385-1393.

Tong, G., and Jahr, C.E. (1994). Multivesicular release from excitatory synapses of cultured hippocampal neurons. Neuron 12, 51-59.

Tsodyks, M.V., and Markram, H. (1997). The neural code between neocortical pyramidal neurons depends on neurotransmitter release probability. Proc. Natl. Acad. Sci. U. S. A. 94, 719-723.

Varela, J.A., Sen, K., Gibson, J., Fost, J., Abbott, L.F., and Nelson, S.B. (1997). A quantitative description of short-term plasticity at excitatory synapses in layer $2 / 3$ of rat primary visual cortex. $\mathrm{J}$. Neurosci. Off. J. Soc. Neurosci. 17, 7926-7940.

Waites, C.L., and Garner, C.C. (2011). Presynaptic function in health and disease. Trends Neurosci. 34, 326-337.

Wang, Y., Gupta, A., Toledo-Rodriguez, M., Wu, C.Z., and Markram, H. (2002). Anatomical, physiological, molecular and circuit properties of nest basket cells in the developing somatosensory cortex. Cereb. Cortex N. Y. N 1991 12, 395-410.

Wang, Y., Markram, H., Goodman, P.H., Berger, T.K., Ma, J., and Goldman-Rakic, P.S. (2006). Heterogeneity in the pyramidal network of the medial prefrontal cortex. Nat. Neurosci. 9, 534-542.

Watanabe, S., Rost, B.R., Camacho-Pérez, M., Davis, M.W., Söhl-Kielczynski, B., Rosenmund, C., and Jorgensen, E.M. (2013). Ultrafast endocytosis at mouse hippocampal synapses. Nature 504, 242247.

Zhang, C., and Peskin, C.S. (2015). Improved signaling as a result of randomness in synaptic vesicle release. Proc. Natl. Acad. Sci. U. S. A. 112, 14954-14959. 


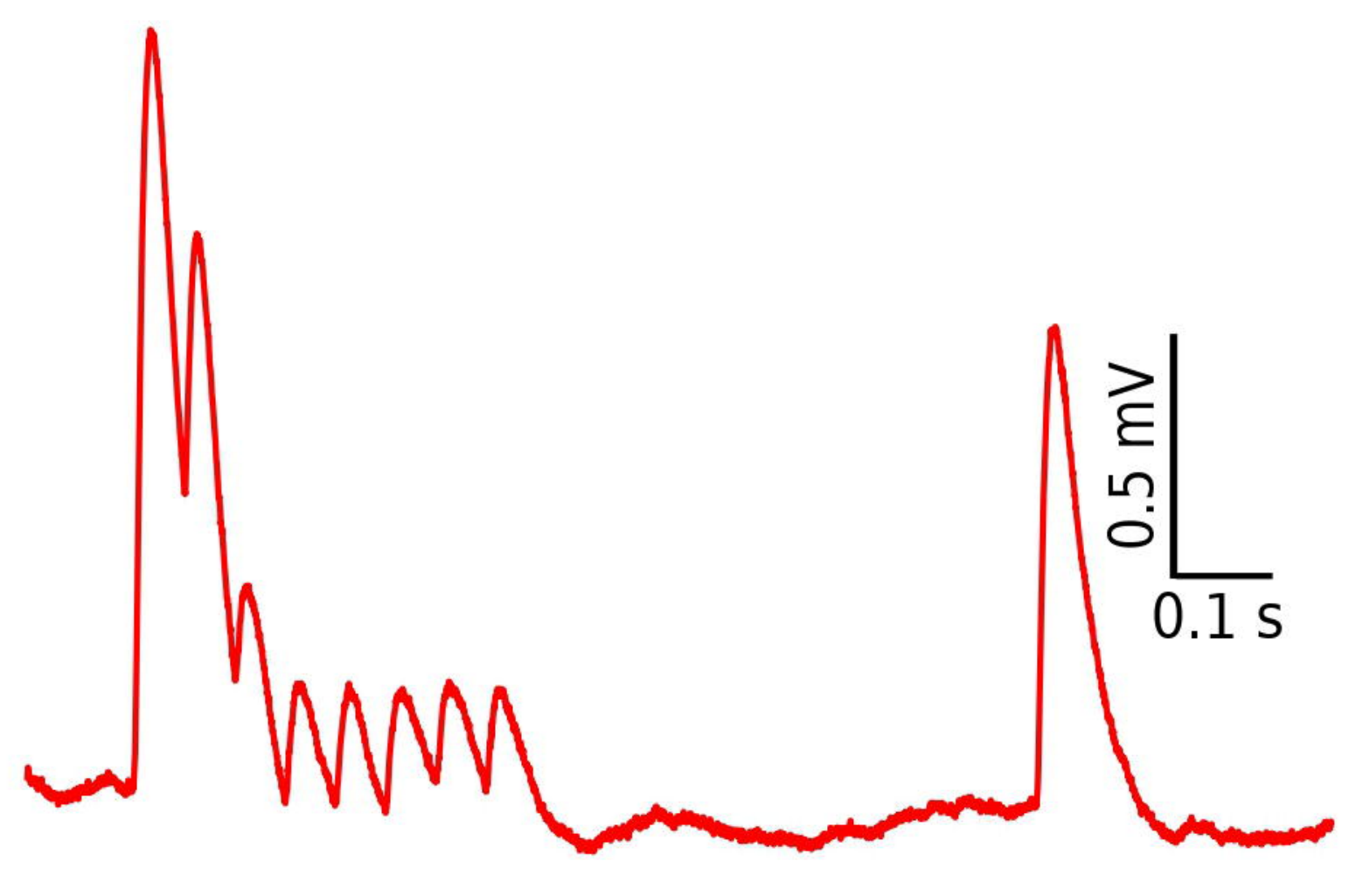

C
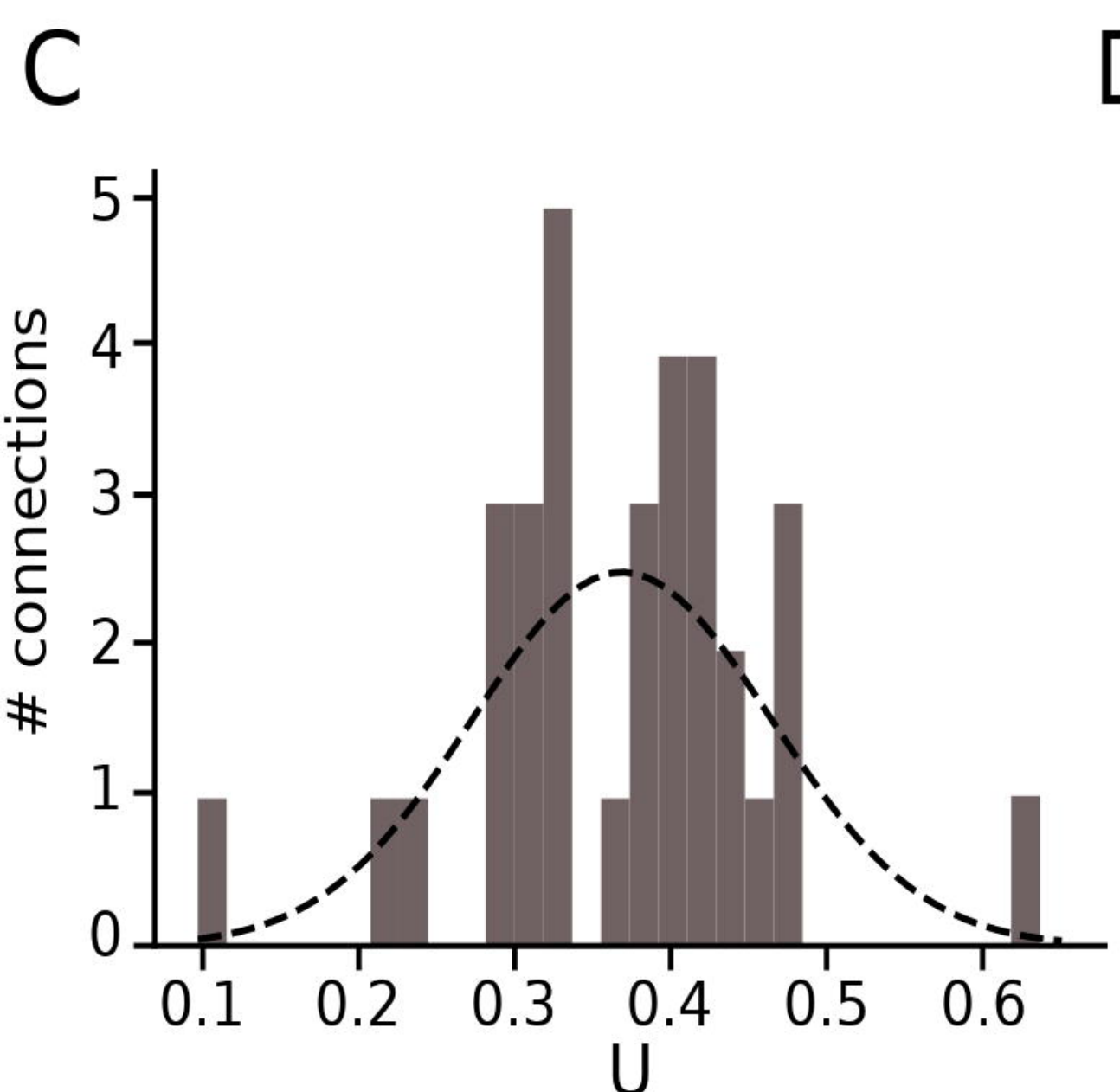

D

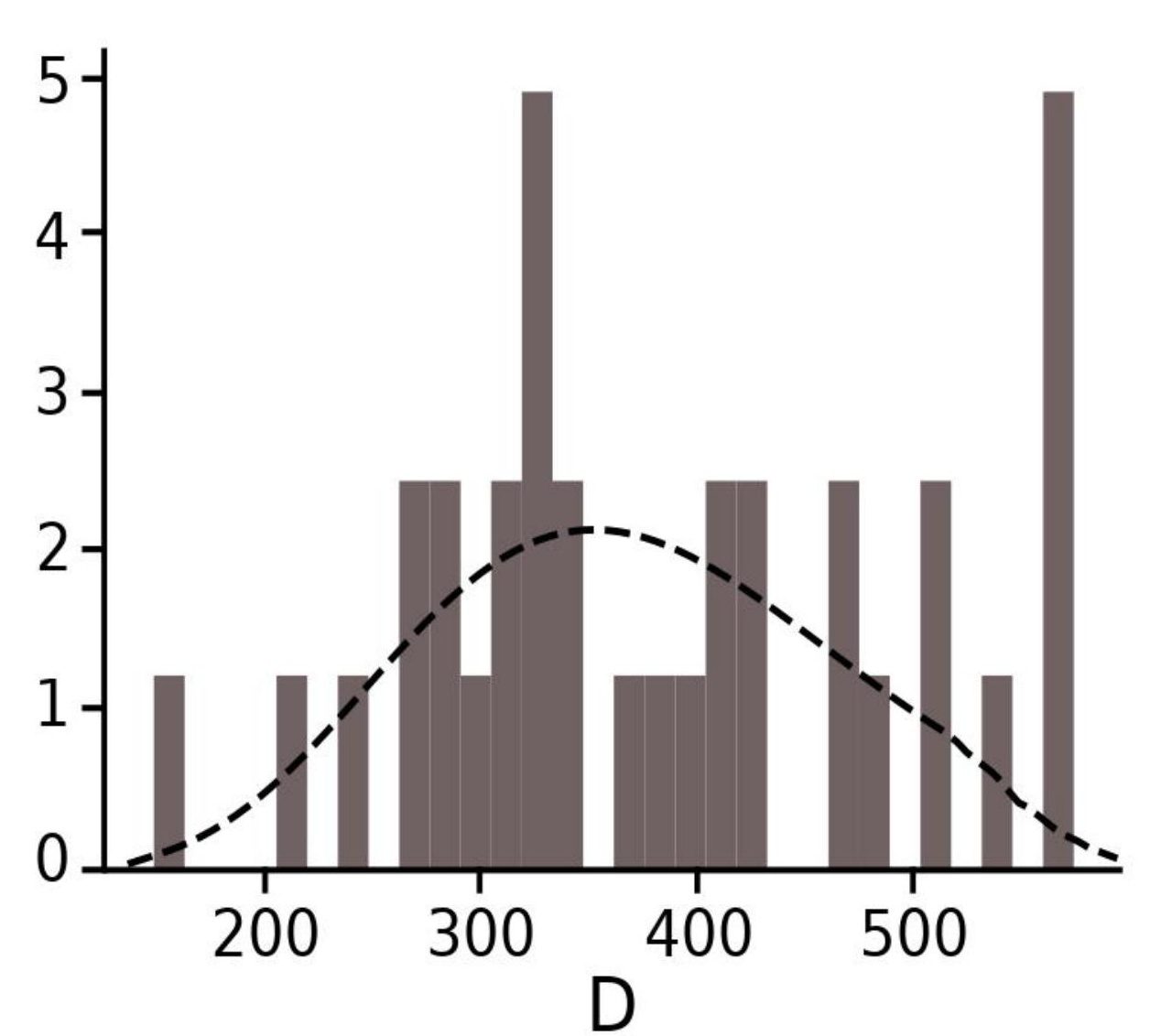

- deconvolved trace

.• . TM-model fitting

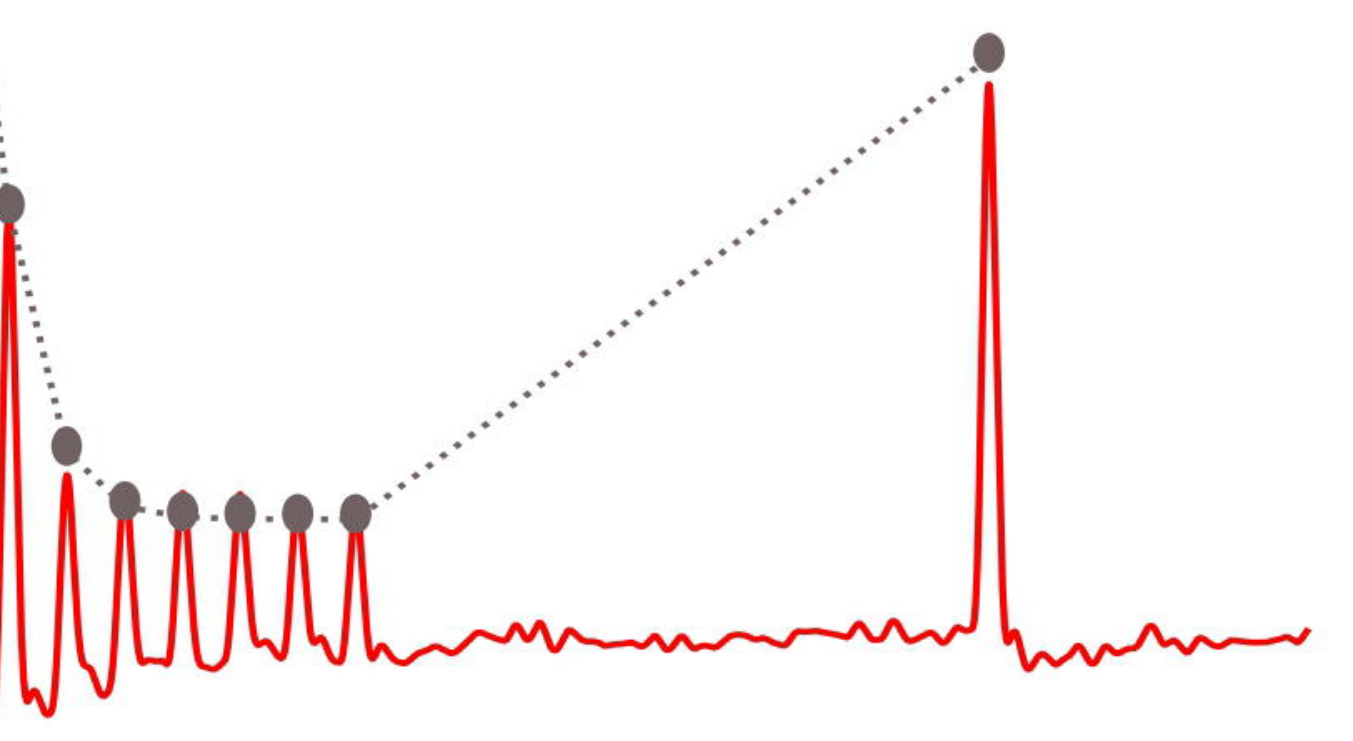

$\mathrm{E}$

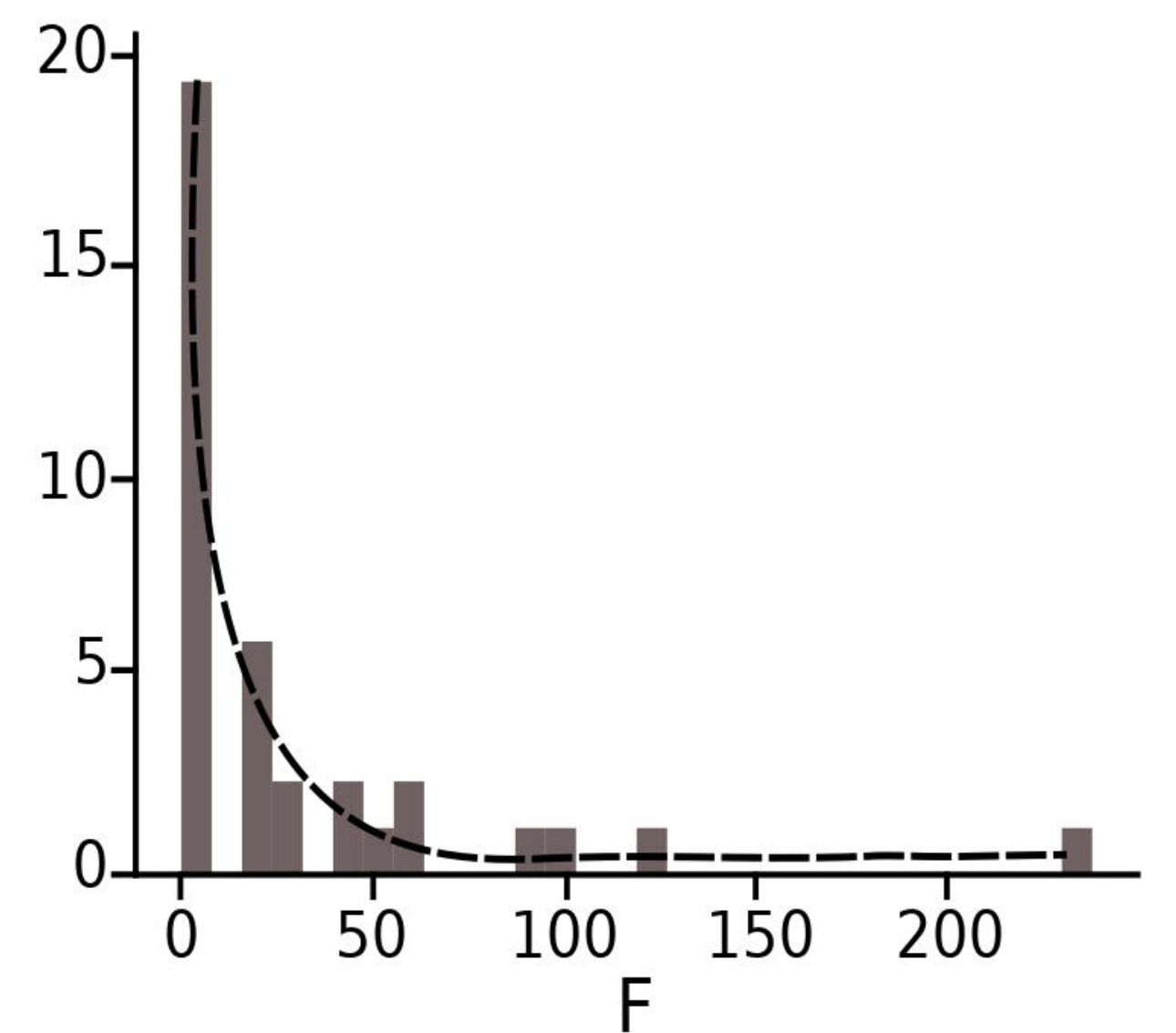




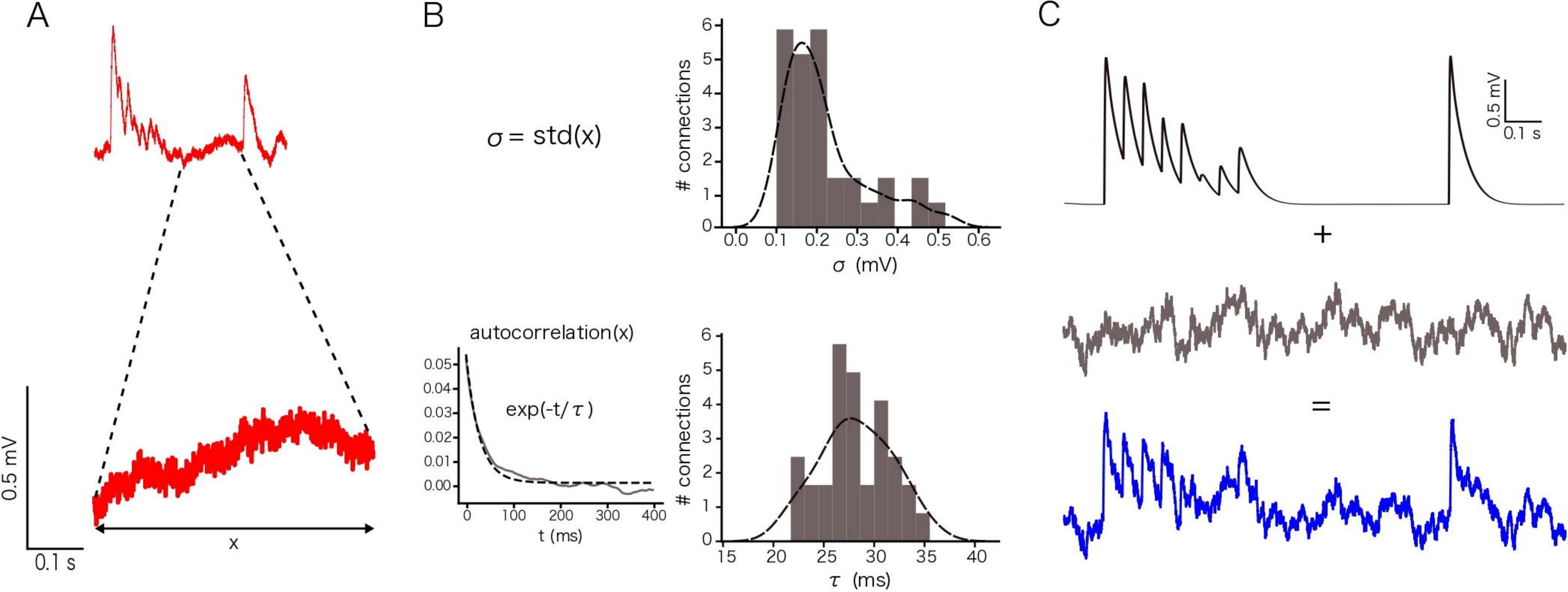




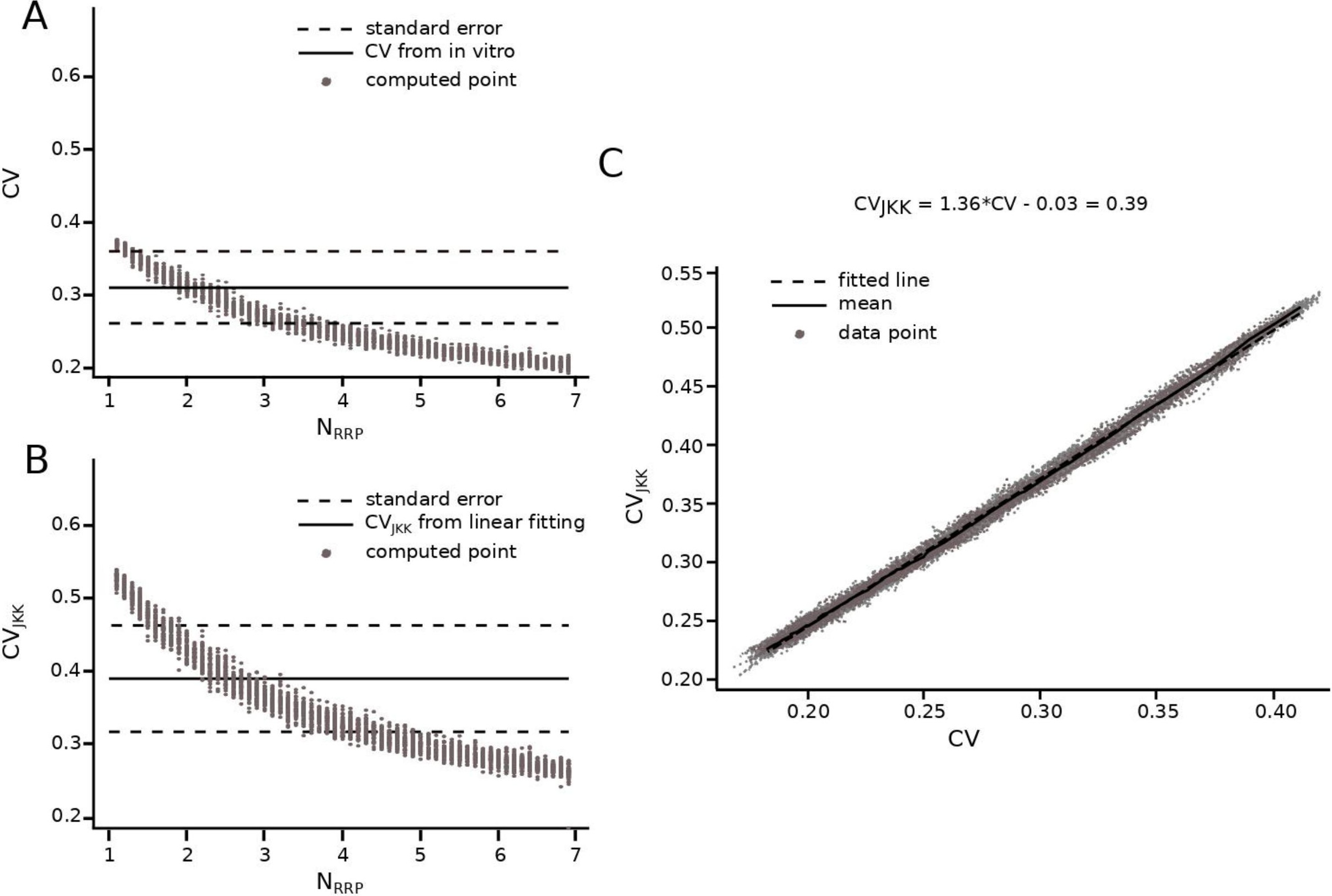

